\title{
Proper orthogonal decomposition of oscillatory Marangoni flow in half-zone liquid bridges of low- $\mathrm{Pr}$ fluids
}

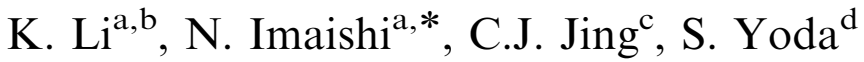 \\ anstitute for Materials Chemistry and Engineering, Kyushu University, 6-1 Kasugakoen, Kasuga 816-8580, Japan \\ binstitute of Mechanics, Chinese Academy of Sciences, Beijing 100080, China \\ ${ }^{\mathrm{c}}$ School of Mechanical Engineering, Southwest Jiaotong University, Chengdu, Sichuan 610031, China \\ ${ }^{\mathrm{d}}$ Institute of Space and Aeronautical Science, Japan Aerospace Exploration Agency, 2-1-1 Sengen, Tsukuba 305-8505, Japan
}

Received 11 July 2006; received in revised form 27 March 2007; accepted 27 March 2007

Communicated by T. Hibiya

Available online 24 May 2007

\begin{abstract}
Proper orthogonal decomposition (POD) using method of snapshots was performed on three different types of oscillatory Marangoni flows in half-zone liquid bridges of low- $P r$ fluid $(P r=0.01)$. For each oscillation type, a series of characteristic modes (eigenfunctions) have been extracted from the velocity and temperature disturbances, and the POD provided spatial structures of the eigenfunctions, their oscillation frequencies, amplitudes, and phase shifts between them. The present analyses revealed the common features of the characteristic modes for different oscillation modes: four major velocity eigenfunctions captured more than $99 \%$ of the velocity fluctuation energy form two pairs, one of which is the most energetic. Different from the velocity disturbance, one of the major temperature eigenfunctions makes the dominant contribution to the temperature fluctuation energy. On the other hand, within the most energetic velocity eigenfuction pair, the two eigenfunctions have similar spatial structures and were tightly coupled to oscillate with the same frequency, and it was determined that the spatial structures and phase shifts of the eigenfunctions produced the different oscillatory disturbances. The interaction of other major modes only enriches the secondary spatio-temporal structures of the oscillatory disturbances. Moreover, the present analyses imply that the oscillatory disturbance, which is hydrodynamic in nature, primarily originates from the interior of the liquid bridge.
\end{abstract}

(C) 2007 Elsevier B.V. All rights reserved.

PACS: $02.70 . \mathrm{Bf} ;$ 44.10. +i; 44.25. +f; 47.20.Dr; 47.54. $+\mathrm{r}$

Keywords: A1. Computer simulation; A1. Fluid flows; A1. Half-zone liquid bridge; A1. Marangoni flow; A1. Oscillatory flow; A2. Microgravity condition; B2. Low-Prandtl number fluid

\section{Introduction}

For decades, extensive studies, e.g. see Ref. [1], have been devoted to the oscillatory Marangoni flow in half-zone liquid bridges of low- $P r$ fluids due to its relation in the technological floating-zone method of crystal growth. Among the research works, limited space experimental studies, e.g. Refs. [2,3], have been reported due to the difficulties in conducting well-controlled experiments induced by the opacity, reactivity, and high melting

\footnotetext{
${ }^{*}$ Corresponding author. Tel.: + 81925837793 ; fax: +81925837796 .

E-mail address: imaishi@cm.kyushu-u.ac.jp (N. Imaishi).
}

temperature of low- $P r$ fluids (mostly liquid metals). On the other hand, since the first prediction through the numerical study conducted by Rupp et al. [4], theoretical studies including both linear instability analyses, e.g. Ref. [5], and direct numerical simulations, e.g. Refs [4-8], have confirmed that Marangoni flow in nonisothermal liquid bridges of low- $P r$ fluids becomes oscillatory via a two-step bifurcation; this implies that the flow is steady and axisymmetric when the imposed temperature difference between the liquid-bridge supports $(\Delta T)$ is small. As $\Delta T$ increases, at the first critical value of $\Delta T\left(\Delta T_{\mathrm{cl}}\right)$, its spatial symmetry breaks down and a three-dimensional steady flow arises; this is referred as stationary bifurcation. As $\Delta T$ 


\section{Nomenclature}

As $=L / R$ aspect ratio

$A_{i}^{m} \quad$ eigenvector for velocity

$A_{i}^{\prime m} \quad$ normalized eigenvector for velocity

$a_{i}(\tau)$ time-dependent coefficient of characteristic mode

$B_{i}^{m} \quad$ eigenvector for temperature

$B_{i}^{\prime m} \quad$ normalized eigenvector for temperature

$b_{i}(\tau) \quad$ coefficient of characteristic mode

$C_{m, n}^{V} \quad$ time-correlation matrix for velocity

$C_{m, n}^{\Theta} \quad$ time-correlation matrix for temperature

$D$ flow domain and integral domain

$L \quad$ liquid-bridge length

$M \quad$ number of the important modes

$m \quad$ azimuthal wave number

$N \quad$ number of snapshots

$T$ temperature

$T_{\mathrm{h}} \quad$ temperature of hot end

$T_{\mathrm{c}} \quad$ temperature of cold end

$\Delta T=\left(T_{\mathrm{h}}+T_{\mathrm{c}}\right) / 2$ temperature scale

$T_{0}=R / V_{0}$ time scale

$P_{\mathrm{L}} \quad$ long-axis symmetry plane of steady flow $(m=2)$

$P_{\mathrm{S}} \quad$ short-axis symmetry plane of steady flow $(m=2)$

$P_{\mathrm{SY}} \quad$ symmetry plane of steady flow $(m=1)$

$\operatorname{Pr}=v / \alpha$ Prandtl number

$p_{0}=\rho V_{0}^{2}$ pressure scale

$p \quad$ dimensionless pressure
$R \quad$ liquid-bridge radius and length scale

$R e=\left|\sigma_{\mathrm{T}}\right| \Delta T R / \rho v^{2}$ capillary Reynolds number

$\mathbf{x}=(r, \theta, z)$ cylindrical coordinate system

$V_{0}=\left|\sigma_{\mathrm{T}}\right| \Delta T / \mu$ velocity scale

$V=\left(V_{r}, V_{\theta}, V_{z}\right)$ dimensionless velocity vector

\section{Greek letters}

$\tau \quad$ dimensionless time

$\tau_{0} \quad$ quasi-steady oscillation period

$\Theta=\left(T-T_{m}\right) / \Delta T$ dimensionless temperature

$v \quad$ kinematic viscosity

$\rho \quad$ density

$\alpha \quad$ thermal diffusivity

$\sigma_{\mathrm{T}} \quad$ temperature coefficient of surface tension

$\lambda$ eigenvalue

$\Phi \quad$ normalized eigenfunction

$\varepsilon=\left(R e-R e_{\mathrm{c} 2}\right) / R e_{\mathrm{c} 2}$ normalized distance from $R e_{\mathrm{c} 2}$

\section{Superscripts}

$m, n \quad m$ th, $n$th component

$V \quad$ velocity related

$\Theta \quad$ temperature related

\section{Subscripts}

$i, j \quad i$ th, $j$ th component

$m, n \quad m$ th, $n$th component

$r, \theta, z \quad r, \theta$, and $z$ directions increases further, at the second critical value of $\Delta T\left(\Delta T_{\mathrm{c} 2}\right)$, a time-dependent three-dimensional flow sets in. Recently, a numerical benchmark work [9] concerning the first bifurcation of the Marangoni flow in liquid bridges of low- $P r$ fluids was devoted jointly by nine groups from several countries. However, the corresponding benchmark results on the second bifurcation to oscillatory Marangoni flow were not available in Ref. [9].

In our previous numerical studies [10-13], the general features of the oscillatory Marangoni flow in liquid bridges of low- $P r$ fluids in microgravity were systematically investigated over a wide range of aspect ratios (As) from 0.6 to 2.2 , and the corresponding stability map for low- $P r$ fluid was proposed [13]. The results indicated that the critical azimuthal wave number $(m)$ of the steady threedimensional Marangoni flow depends strongly on As, e.g. $m=1$ in long liquid bridges of As $=2.0, m=2$ in intermediate liquid bridges of $\mathrm{As}=1.4$ and 1.0, and $m=3$ in shorter liquid bridges of As $=0.6$. These results qualitatively agree with the empirical correlation [1], namely, $m \approx 2 /$ As. For the oscillatory Marangoni flow at slightly supercritical conditions, the flow field $F(\mathbf{x}, \tau)$ can be characterized as the superposition of the time-dependent three-dimensional velocity or temperature disturbances
$F^{\prime}(\mathbf{x}, \tau)$ onto time-averaged steady (basic) fields $\bar{F}(\mathbf{x}): F(\mathbf{x}, \tau)=\bar{F}(\mathbf{x})+F^{\prime}(\mathbf{x}, \tau)$. The basic fields have the same characteristic azimuthal wave number $(m)$ as the corresponding three-dimensional steady flow, and the timedependent velocity and temperature disturbances may exhibit complex behaviors. However, the detailed spatiotemporal structures of the oscillatory velocity and temperature disturbances of Marangoni flows in liquid bridges of low- $P r$ fluids remain largely unknown.

Proper orthogonal decomposition (POD) analysis [14] is a rigorous procedure for extracting a basis of characteristic modes from sampled time evolution signals. These modes are the eigenfunctions of an integral operator based on the spatial correlation function. They are shown to form an orthogonal basis for the function space in which the process resides and to represent this process in the most efficient way. However, the direct application of POD to a discretized three-dimensional flow problem involves extremely heavy computational tasks because the dimension of the spatial correlation matrix corresponds to the mesh number in the direct numerical simulation. A more accessible approach is the method of snapshots [15]. This method, which invokes the ergodic hypothesis, can reduce the computational task to a more tractable eigenproblem 
of a size equal to the snapshot number of the flow field obtained through direct numerical simulation. An overview of POD and its applications can be found in Refs. [16,17]. However, POD analyses on the oscillatory Marangoni flow in liquid-bridge configurations are still lacking.

In the present study, the POD analysis using the method of snapshots was applied to the oscillatory Marangoni flows in half-zone liquid bridges of $P r=0.01$ and As $=1.0$, 1.4 , and 2.0 at certain Reynolds numbers. A series of characteristic modes and their temporal expansion coefficients were extracted from the oscillatory velocity and temperature disturbances. Through the analyses of the spatial structures of the characteristic modes and their temporal behaviors, the present study aims to obtain the details of the spatio-temporal structures of the threedimensional oscillatory velocity and temperature disturbances.

\section{Problem statement}

Fig. 1 shows the schematics of a typical half-zone liquid bridge model adopted in the present study. The adiabatic melt-free surface is assumed to be nondeformable and cylindrical in a microgravity condition. The origin of the cylindrical coordinate is located at the center of the lower disc. The fluid with $P r=0.01$ is representative of typical semiconductor melts or liquid metals and is assumed to be an incompressible Newtonian fluid with constant properties. The corresponding fundamental equations are expressed in a dimensionless form as follows:

$\nabla \cdot V=0$

$\frac{\partial V}{\partial \tau}+V \cdot \nabla V=-\nabla p+\frac{1}{R e} \nabla^{2} V$

$\frac{\partial \Theta}{\partial \tau}+V \cdot \nabla \Theta=\frac{1}{\operatorname{RePr}} \nabla^{2} \Theta$

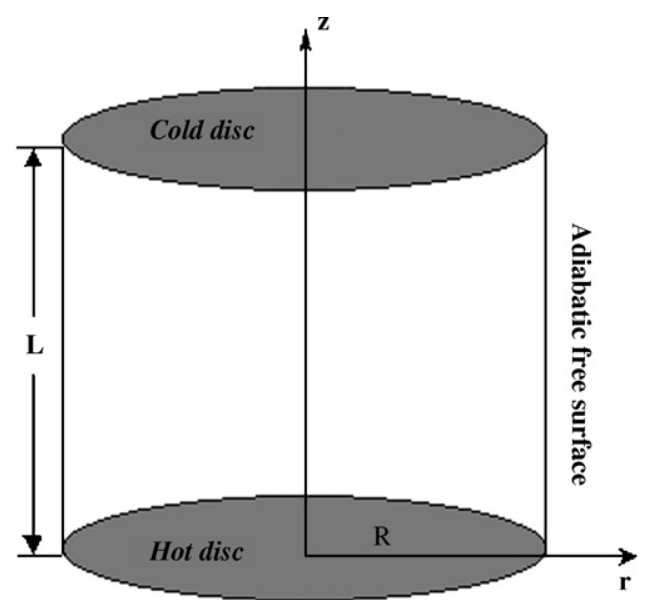

Fig. 1. Schematics of the half-zone liquid bridge model.
The boundary conditions are as follows:

At the free surface $(r=1)$ :

$V_{r}=0, \quad r \frac{\partial}{\partial r}\left(\frac{V_{\theta}}{r}\right)=-\frac{1}{r} \frac{\partial \Theta}{\partial \theta}, \quad \frac{\partial V_{z}}{\partial r}=-\frac{\partial \Theta}{\partial z}, \quad \frac{\partial \Theta}{\partial r}=0$.

At the upper cold disc $(z=\mathrm{As})$ :

$V=0, \quad \Theta=-0.5$.

At the lower hot disc $(z=0)$ :

$V=0, \quad \Theta=0.5$.

The fundamental equations are discretized by the finite control-volume method based on a staggered grid.
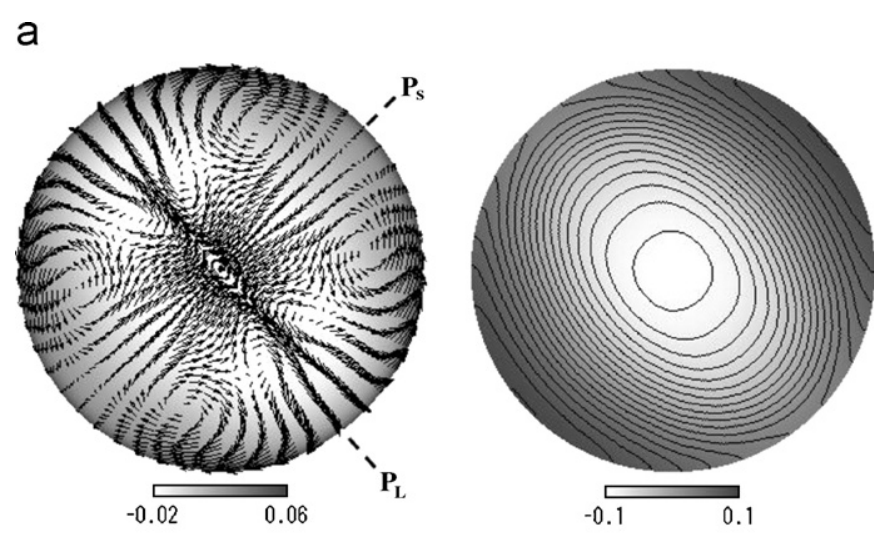

b
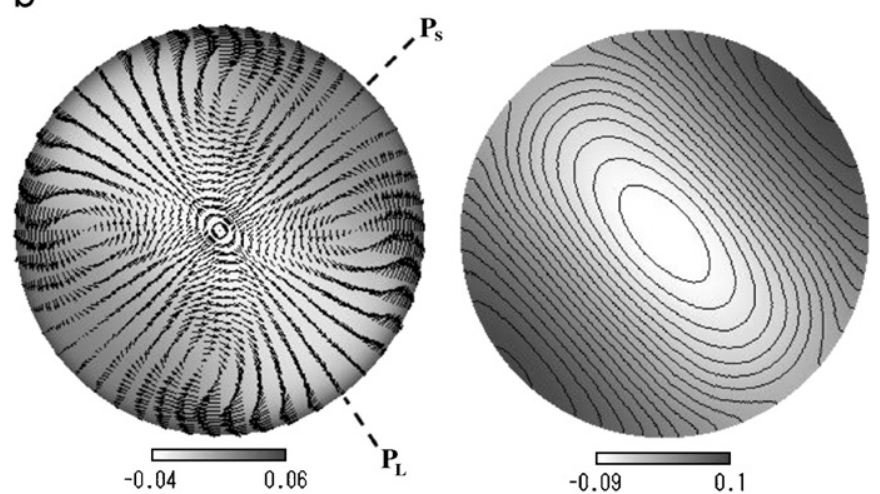

C
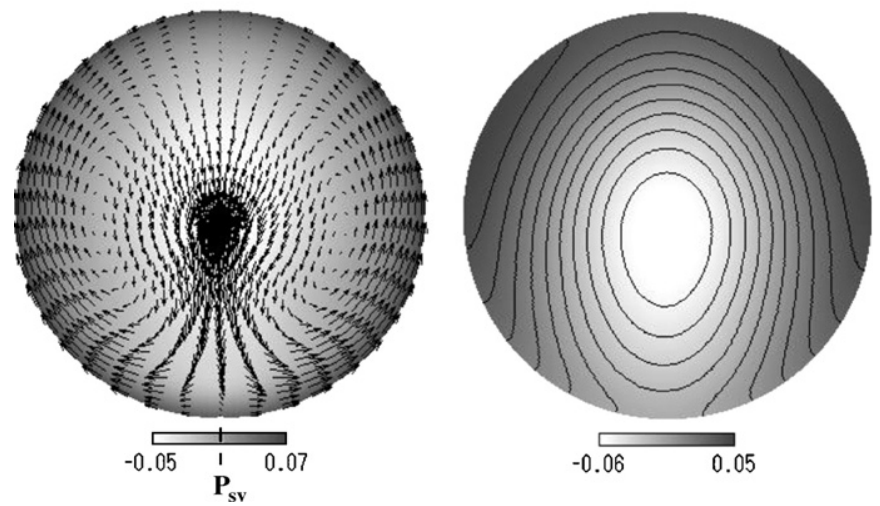

Fig. 2. Projections of basic flow and thermal fields onto the $z=0.5 \mathrm{As}$ plane: (a) $2 P$ oscillatory flow, (b) $2 T$ oscillatory flow and (c) $1 T$ oscillatory flow (gray color in the flow-field plots denotes the axial velocity). 
a

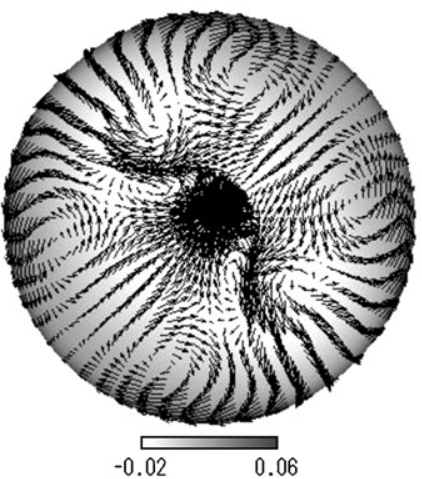

b

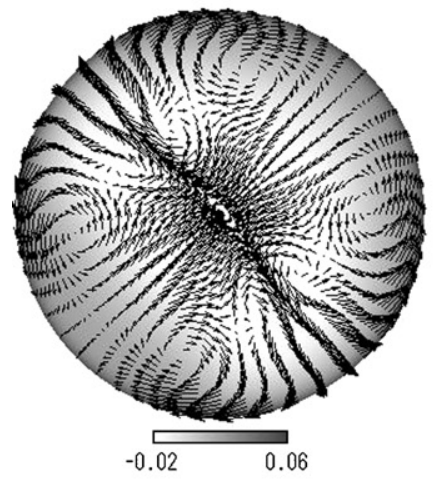

C

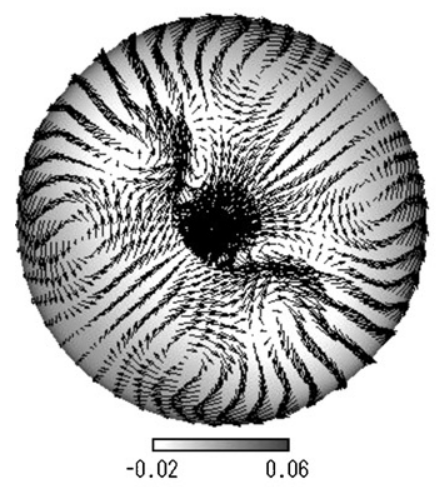

d
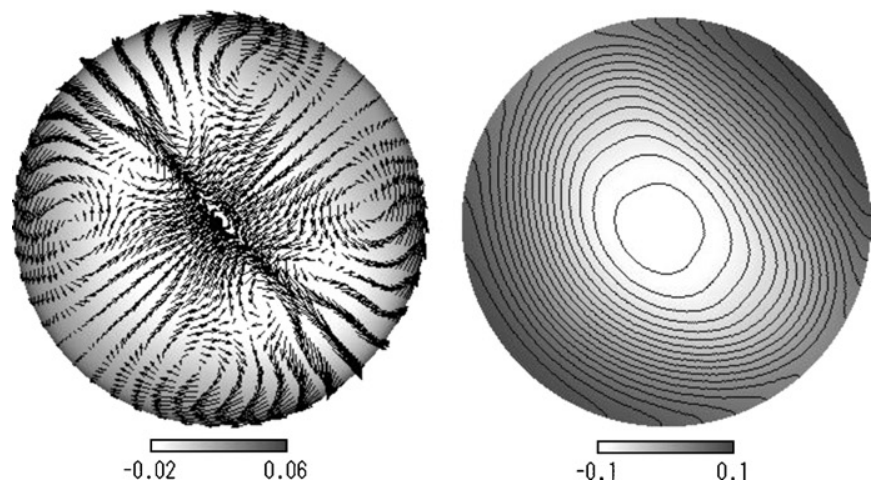

Fig. 3. Projections of flow and thermal fields of $2 P$ oscillatory flow onto the $z=0.5 \mathrm{As}$ plane for a quasi-steady oscillation period at intervals of $\tau_{0} / 4$ (gray color in the flow-field plots denotes the axial velocity). a
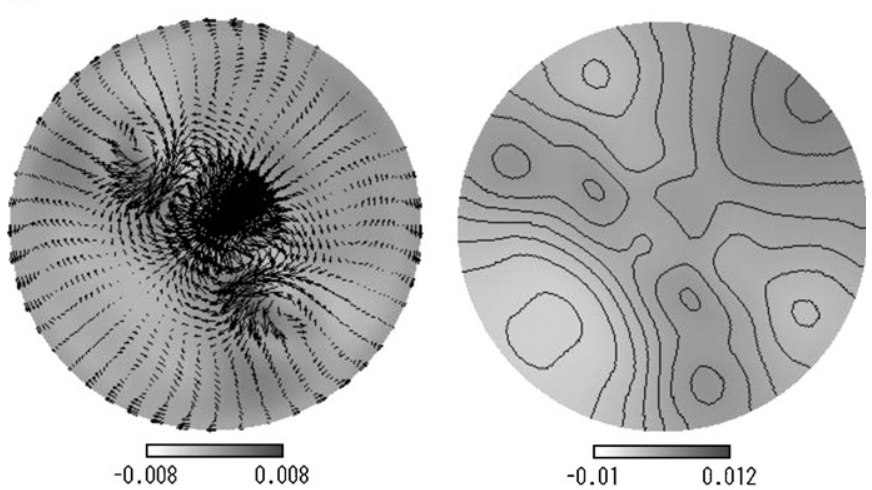

b
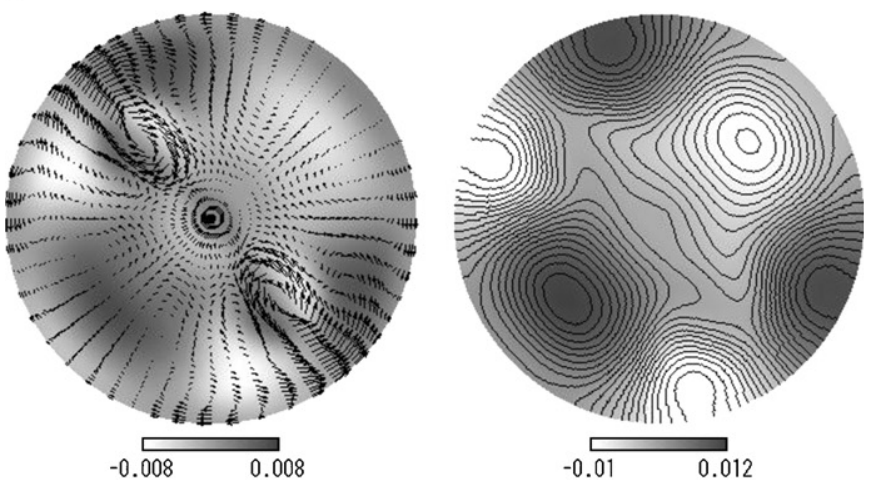

C
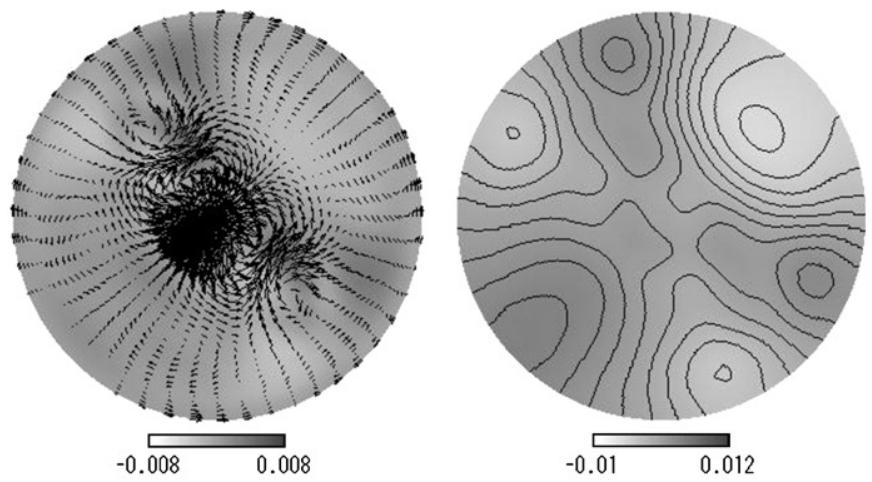

d
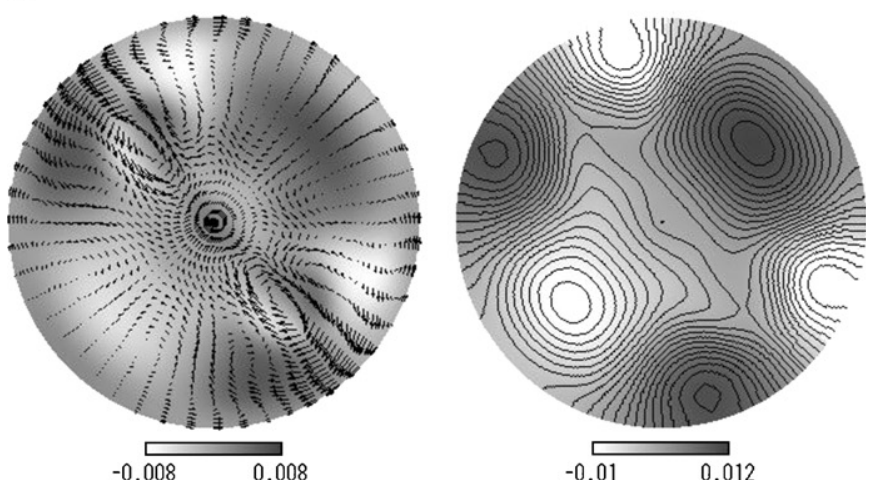

Fig. 4. Projections of velocity and temperature disturbances of the $2 P$ oscillatory flow onto the $z=0.5$ As plane for a quasi-steady oscillation period at intervals of $\tau_{0} / 4$ (gray color in the flow-field plots denotes the axial velocity). 
Nonuniform grids in radial and axial directions are adopted to increase the resolution near the boundary. The details of the numerical methods were described in Refs. [10-13] and the code validation can be found in Ref. [10].

\section{POD using the method of snapshots}

The practical procedures for POD using the method of snapshots that was applied in the present study are described here.

The primary variables $V(\mathbf{x}, \tau)$ and $\Theta(\mathbf{x}, \tau)$ obtained through the $N$ snapshots are decomposed into timeaveraged parts, $\bar{V}(\mathbf{x})$ and $\bar{\Theta}(\mathbf{x})$, and time-varying parts, $V^{\prime}(\mathbf{x}, \tau)$ and $\Theta^{\prime}(\mathbf{x}, \tau)$, i.e.,

$V(\mathbf{x}, \tau)=\bar{V}(\mathbf{x})+V^{\prime}(\mathbf{x}, \tau), \quad \Theta(\mathbf{x}, \tau)=\bar{\Theta}(\mathbf{x})+\Theta^{\prime}(\mathbf{x}, \tau)$.

The two time-correlation matrices $C_{m, n}^{V}$ and $C_{m, n}^{\Theta}$ are then constructed from the velocity and temperature samples, respectively, as follows:

$C_{m, n}^{V}=\frac{1}{N}\left(V^{\prime}\left(\mathbf{x}, \tau_{m}\right), V^{\prime}\left(\mathbf{x}, \tau_{n}\right)\right), \quad m, n=1,2, \ldots, N$,

$C_{m, n}^{\Theta}=\frac{1}{N}\left(\Theta^{\prime}\left(\mathbf{x}, \tau_{m}\right), \Theta^{\prime}\left(\mathbf{x}, \tau_{n}\right)\right), \quad m, n=1,2, \ldots, N$.

Here, the outer parentheses represent the inner product; the velocity and temperature are treated separately during the construction of the time-correlation matrices [18-20].

The eigenvalues $\lambda_{i}^{V}$ and $\lambda_{i}^{\Theta}$ associated with the above matrices and their corresponding eigenvectors $A_{i}^{m}$ and $B_{i}^{m}$ can be calculated, which gives

$C_{m, n}^{V} A_{i}^{n}=\lambda_{i}^{V} A_{i}^{m}, \quad C_{m, n}^{V} A_{i}^{n}=\lambda_{i}^{V} A_{i}^{m}, \quad m=1,2, \ldots, N$.

Finally, the characteristic modes (also known as empirical eigenfunctions) $\Phi_{i}^{V}(\mathbf{x})$ and $\Phi_{i}^{\Theta}(\mathbf{x})$ (here normalized) are obtained as linear combinations of the time-varying parts

$\Phi_{i}^{V}(\mathbf{x})=\sum_{m=1}^{N} A_{i}^{\prime m} V^{\prime}\left(\mathbf{x}, \tau_{m}\right), \quad \Phi_{i}^{\Theta}(\mathbf{x})=\sum_{m=1}^{N} B_{i}^{\prime m} \Theta^{\prime}\left(\mathbf{x}, \tau_{m}\right)$

with $A_{i}^{\prime m}=A_{i}^{m} / \sqrt{\lambda_{i}^{V} N \sum_{m=1}^{N}\left(A_{i}^{\prime m}\right)^{2}} \quad$ and $\quad B_{i}^{\prime m}=B_{i}^{m} /$ $\sqrt{\lambda_{i}^{\Theta} N \sum_{m=1}^{N}\left(B_{i}^{\prime m}\right)^{2}}$.

On the other hand, the time-varying parts $V(\mathbf{x}, \tau)$ and $\Theta(\mathbf{x}, \tau)$ can be reproduced in terms of these characteristic modes as

$v^{\prime}(\mathbf{x}, \tau)=\sum_{i=1}^{M_{V}} \boldsymbol{a}_{i}(\tau) \Phi_{i}^{V}(\mathbf{x}), \quad \Theta^{\prime}(\mathbf{x}, \tau)=\sum_{i=1}^{M_{\Theta}} \boldsymbol{b}_{i}(\tau) \Phi_{i}^{\Theta}(\mathbf{x})$,

where $\boldsymbol{a}_{i}(\tau)$ and $\boldsymbol{b}_{i}(\tau)$ are time-dependent coefficients; $M_{V}$ and $M_{\Theta}$ are the numbers of characteristic modes retained in the expansion for velocity and temperature, respectively. Usually, $M_{V}$ and $M_{\Theta}$ are much smaller than the snapshot number $N$, and the accuracy of the reconstruction can be improved by increasing $M_{V}$ and $M_{\Theta}$. When $M_{V}=M_{\Theta}=$ $N$, the original sampled signals can be reproduced exactly, with $\boldsymbol{a}_{i}(\tau)=N \lambda_{i}^{V} A_{i}^{\prime m}$ and $\boldsymbol{b}_{i}(\tau)=N \lambda_{i}^{T} B_{i}^{\prime m}$.

\section{Results}

In our previous numerical studies [10-13], we reported three types of oscillatory velocity and temperature disturbances, i.e., " $P$ ", " $T$ ", and " $R$ ", which were classified based on the time-dependent deformations of flow and thermal fields in the midplane of the liquid bridge. " $P$ " represents the disturbance exhibiting a pendulum-like pulsating motion across the axis of the liquid bridge, " $T$ " represents the disturbance accompanying the twisting (or torsional) motion of one of the symmetry axes of the basic fields, and " $R$ " represents the bulk rotational motion of the basic fields about the axis of the liquid bridge. Thus, the

Table 1

Eigenvalues of major characteristic modes of velocity and temperature disturbances for $\operatorname{Pr}=0.01$, As $=1.0$, and $R e=8000$

\begin{tabular}{|c|c|c|c|c|}
\hline Eigenfunction, $i$ & \multicolumn{2}{|l|}{ Velocity disturbance } & \multicolumn{2}{|l|}{ Temperature disturbance } \\
\hline 1 & 0.5024 & 50.236 & 0.8879 & 88.787 \\
\hline 2 & 0.4199 & 92.228 & $6.3906 \times 10^{-2}$ & 95.178 \\
\hline 3 & $4.5550 \times 10^{-2}$ & 96.783 & $3.8132 \times 10^{-2}$ & 98.990 \\
\hline 6 & $2.5162 \times 10^{-3}$ & 99.873 & $6.3905 \times 10^{-4}$ & 99.980 \\
\hline 7 & $5.7302 \times 10^{-4}$ & 99.930 & $1.1151 \times 10^{-4}$ & 99.990 \\
\hline 8 & $4.2220 \times 10^{-4}$ & 99.973 & $5.9710 \times 10^{-5}$ & 99.996 \\
\hline 9 & $1.3524 \times 10^{-4}$ & 99.986 & $1.9264 \times 10^{-5}$ & 99.998 \\
\hline 10 & $8.2950 \times 10^{-5}$ & 99.994 & $1.0053 \times 10^{-5}$ & 99.999 \\
\hline
\end{tabular}


corresponding oscillatory Marangoni flows are denoted as the disturbance types combined with the critical azimuthal wave number of the basic fields, e.g. $(2 P),(2 T)$, and $(2 R)$

a
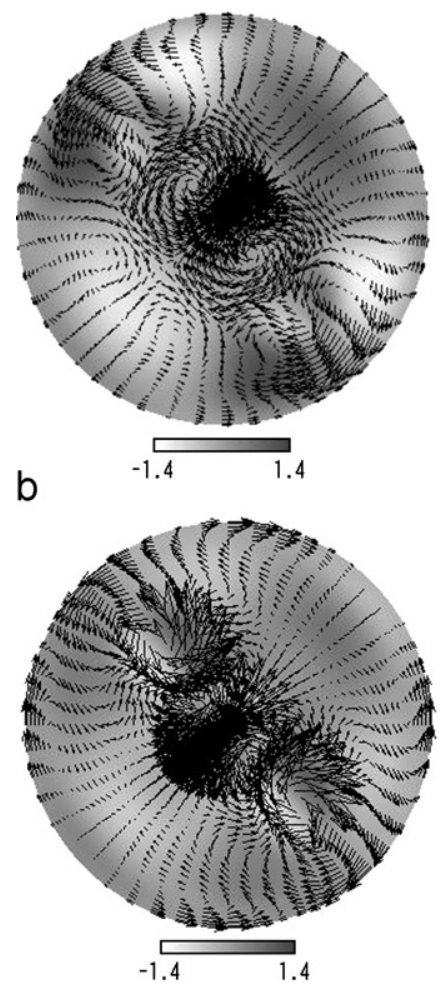

C

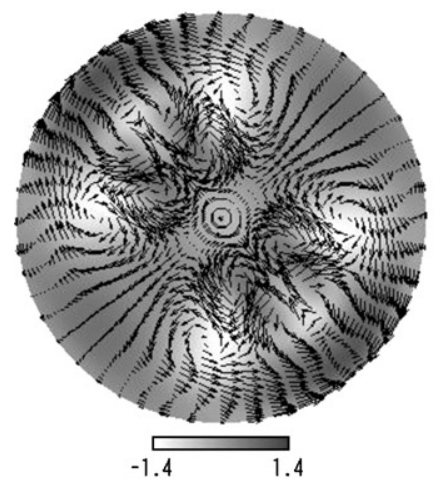

d
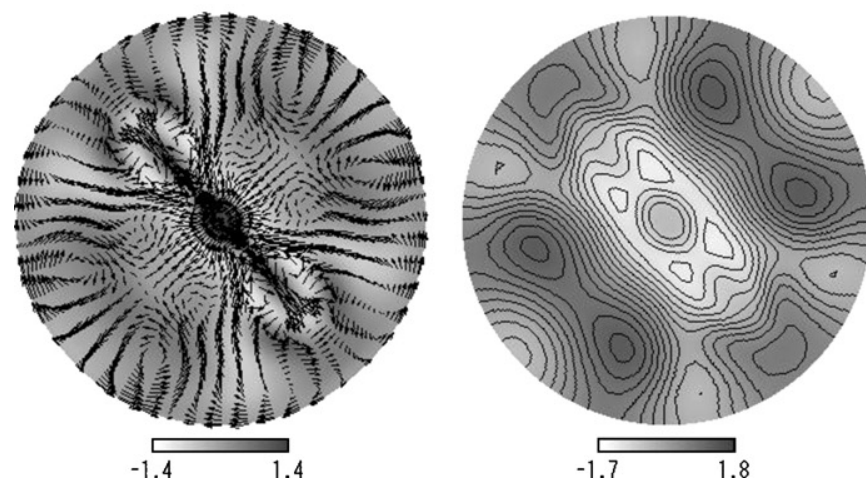

Fig. 5. Projections of the major velocity and temperature characteristic modes of the $2 P$ oscillatory flow onto the $z=0.5 \mathrm{As}$ plane (gray color in the flow-field plots denotes the axial velocity). for $m=2$. The aforementioned characteristics of the oscillatory Marangoni flows are analogous to those in liquid bridges of high- $P r$ fluids. However, the instability mechanism for low- $P r$ fluids is hydrodynamic in nature and fundamentally different from that for high-Pr fluids, which is due to hydrothermal waves [1].

In present study, three cases, i.e., (1) $P r=0.01$, As $=$ 1.0, $R e=8000$; (2) $P r=0.01$, As $=1.4, R e=6500$; and (3) $\operatorname{Pr}=0.01, \mathrm{As}=2.0, R e=3500$, were investigated. Cases 1 and 2 represent liquid bridges of intermediate length, and the third case represents a long liquid bridge. The second critical Reynolds numbers for half-zone liquid bridges of a

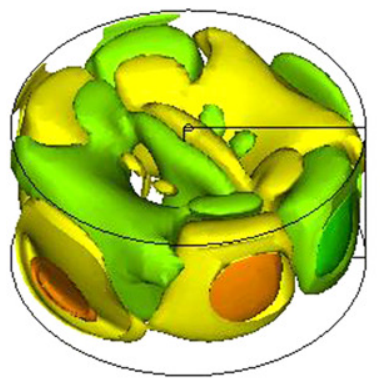

C

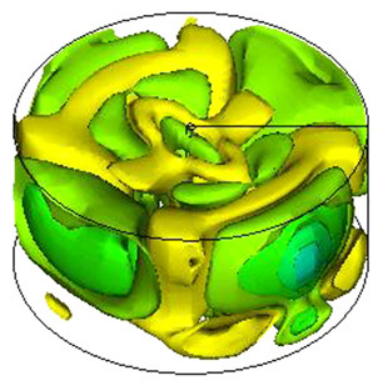

e

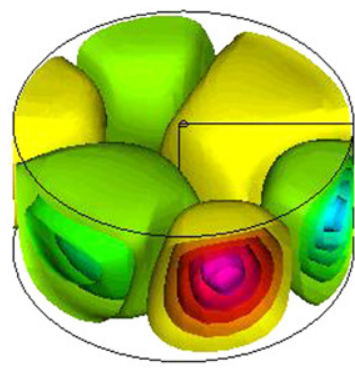

g

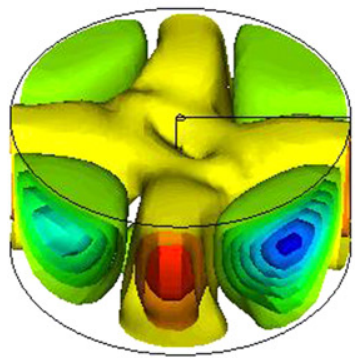

b

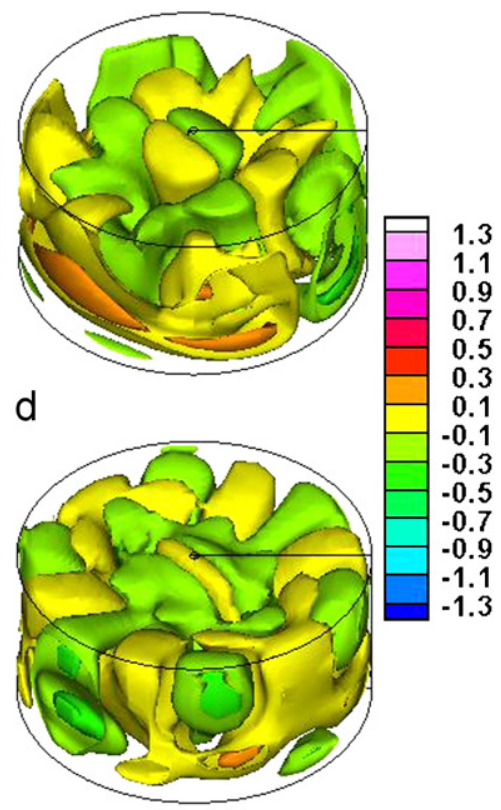

f

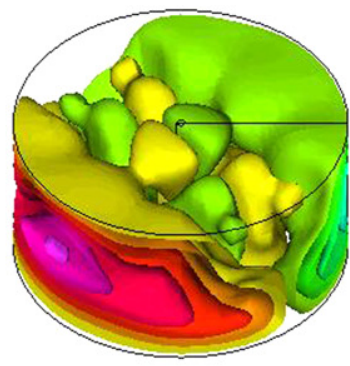

h

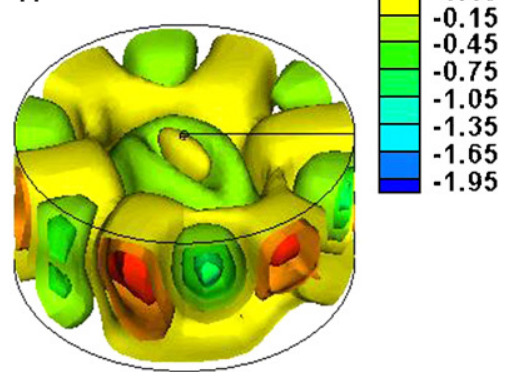

Fig. 6. Iso-surfaces of the major characteristic modes of $2 P$ oscillatory flow: (a)-(d): the velocity characteristic modes and (e)-(h) the temperature characteristic modes. 
$P r=0.01$ are $R e_{\mathrm{c} 2}=6650$ for $\mathrm{As}=1.0, R e_{\mathrm{c} 2}=4628$ for As $=1.4$, and $R e_{\mathrm{c} 2}=2865$ for As $=2.0$ [13]. Thus, the Reynolds number for each case is slightly supercritical, and the corresponding saturated oscillatory Marangoni flow reaches the quasi-steady states (periodic oscillation with constant amplitude). Data sets of the flow and thermal fields covering three periods of the quasi-steady oscillations were selected as the sampled time evolution signals. For each case, totally 120 snapshots were taken from the signal with equal time interval between two successive snapshots. POD analysis using the method of snapshots was conducted on the sampled signals, and the results are described in the following sections.

\subsection{Case 1: $\operatorname{Pr}=0.01, A s=1.0, \operatorname{Re}=8000$, and $\varepsilon=0.2$}

For the present case, the corresponding three-dimensional steady flow is $m=2$ [10], and the flow and thermal fields are mirror symmetric with respect to two orthogonal planes, i.e., the meridian planes consisting of the long and short axes of the saddle-shaped vortex core (denoted by $P_{\mathrm{L}}$ and $P_{\mathrm{S}}$ ) (see Fig. 2a). The saturated quasi-steady oscillatory Marangoni flow is of the $(2 P)$ type. Fig. 3 shows the oscillatory flow and thermal fields in a plane at $z=0.5 \mathrm{As}$ at four different instants during an oscillation period. Following the procedures described in Section 3, the basic flow and thermal fields show the similar characteristics as the three-dimensional steady flow of $m=2$ as shown in Fig. 2a. During the oscillation period, the velocity disturbance moves back and forth along $P_{\mathrm{S}}$ across the axis of the liquid bridge; this disturbance is accompanied by pairs of intense vortices (with every pair having the opposite vorticity with regard to the previous pair) that are annihilated and recreated periodically around $r=0.5$ along $P_{\mathrm{L}}$ (see Fig. 4). Correspondingly, the hot spots and cold spots of the temperature disturbance expand and contract periodically and occupy the same spatial positions alternately. Both the velocity and temperature disturbances show a mirror symmetry with respect to $P_{\mathrm{S}}$, indicating the dominant odd azimuthal wave numbers of the disturbances. It is also noted that on the free surface of the fluid, the azimuthal component of the velocity disturbance is

a

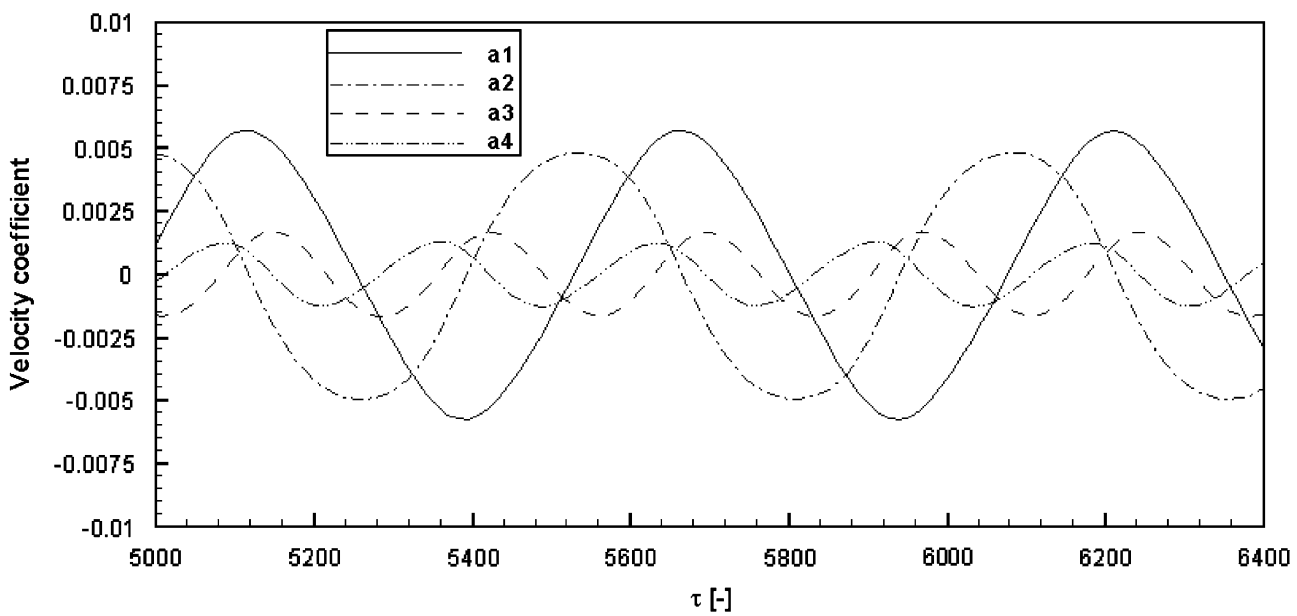

b

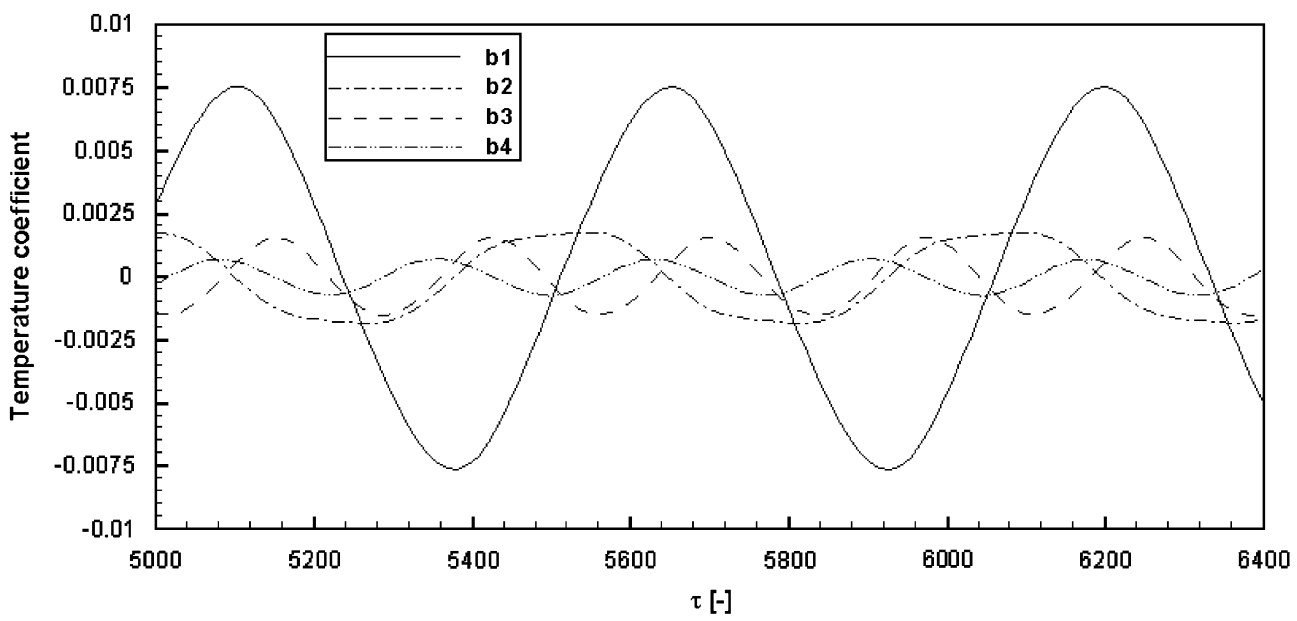

Fig. 7. Temporal expansion coefficients of the major characteristic modes for the $2 P$ oscillation: (a) velocity characteristic modes and (b) temperature characteristic modes. 
a

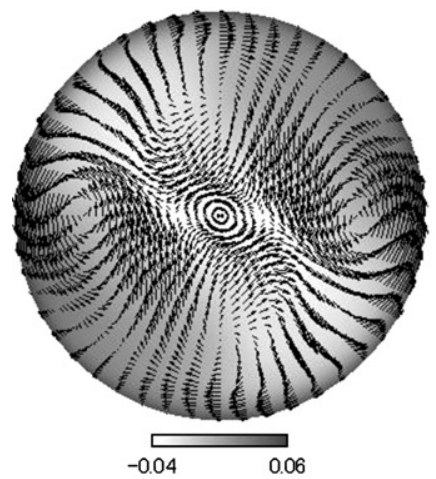

b

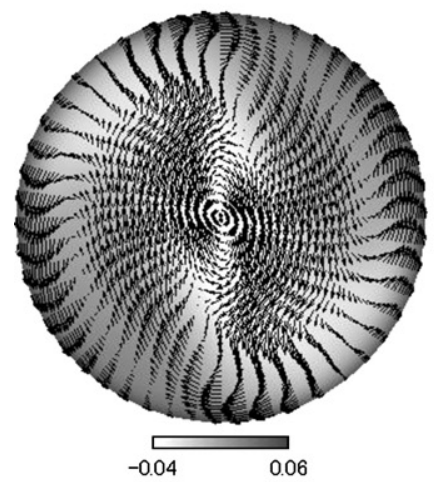

C

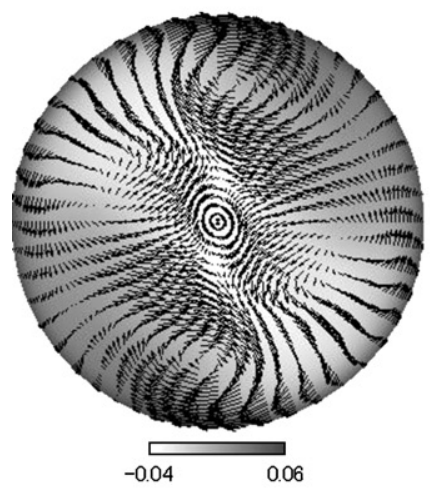

d
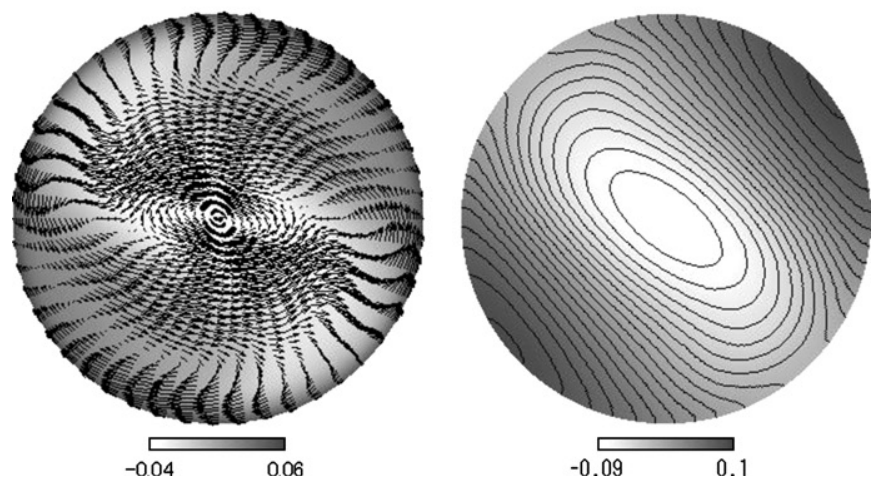

Fig. 8. Projections of flow and thermal fields of the $2 T$ oscillatory flow onto the $z=0.5 \mathrm{As}$ plane for a quasi-steady oscillation period at intervals of $\tau_{0} / 4$ (gray color in the flow-field plots denotes the axial velocity). a
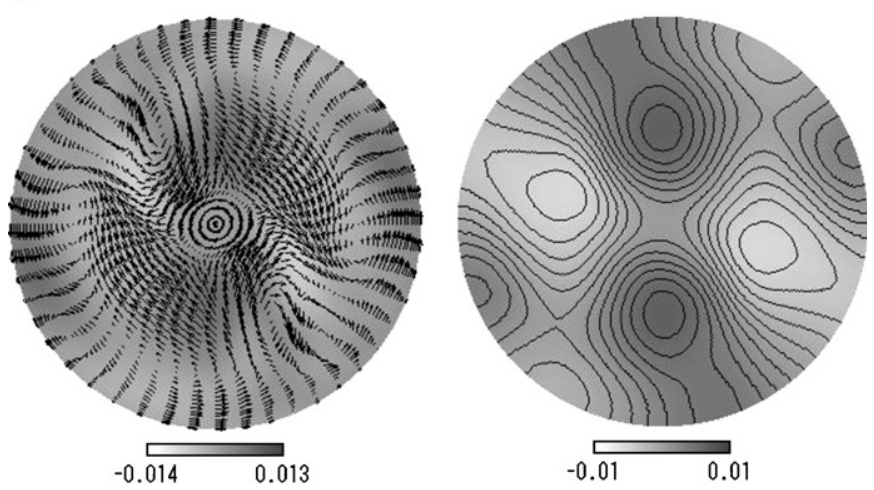

b
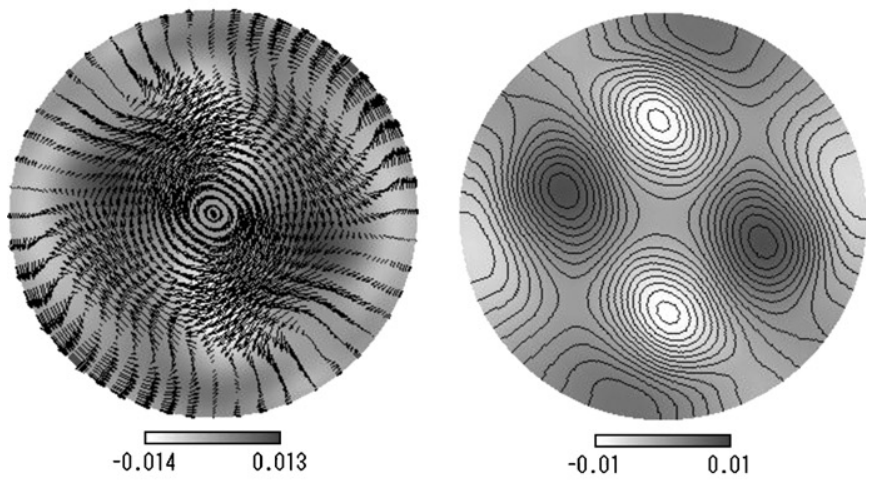

C
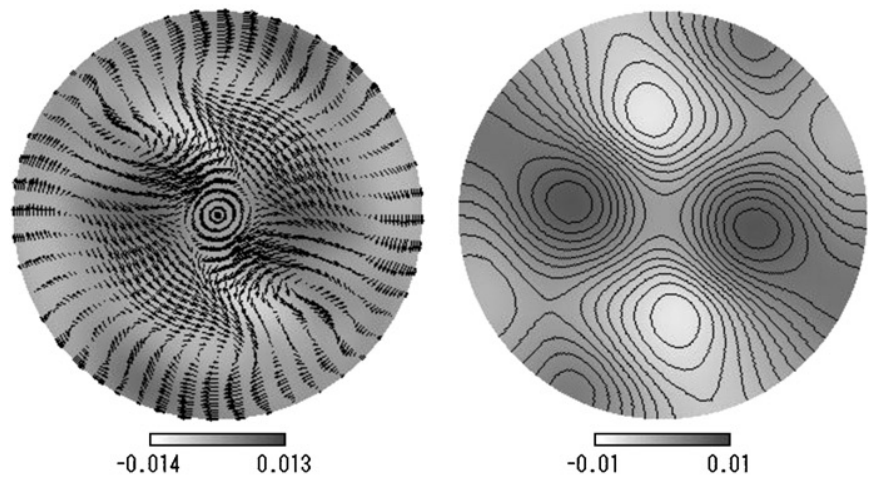

d
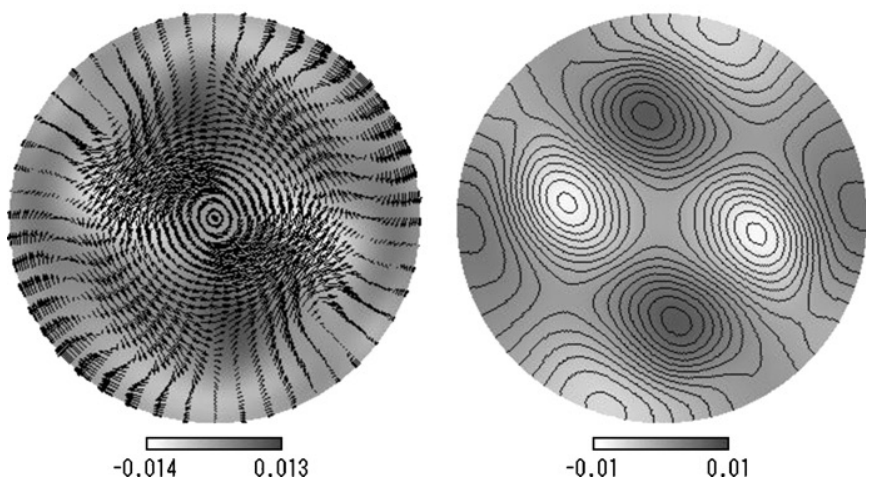

Fig. 9. Projections of velocity and temperature disturbances of the $2 T$ oscillatory flow onto the $z=0.5$ As plane for a quasi-steady oscillation period at intervals of $\tau_{0} / 4$ (gray color in the flow-field plots denotes the axial velocity). 
directed from the cold spots to the hot spots, which reflects the hydrodynamic nature of the instability. The velocity and temperature disturbances can be decomposed into a series of characteristic modes (eigenfunctions) by POD analysis. Table 1 shows the 10 largest eigenvalues normalized by requiring $\sum_{1}^{N} \lambda_{i}=1$ for the velocity and temperature disturbances, respectively; they are real, positive, and ordered by their magnitude which represents the contribution of the corresponding characteristic mode to the velocity (temperature) fluctuation energy [21]. For both velocity disturbance and temperature disturbance, the first four characteristic modes, namely, major modes, totally capture more than $99 \%$ of the fluctuation energy. For the velocity disturbance, the major modes can be divided into two pairs based on the eigenvalue magnitude, and the first pair is the most energetic which captures more than $90 \%$ of the fluctuation energy. The energy distribution among the major modes for the temperature disturbance is different, and the first characteristic mode is the most energetic, which captures about $90 \%$ of the fluctuation energy alone. Figs. 5 and 6 show the major velocity and temperature modes and their spatial structures. Within the most energetic velocity characteristic mode pair, the two modes mirror symmetric to $P_{\mathrm{S}}$ represent the large scale features of the velocity disturbance; they both have strong velocity components parallel to $P_{\mathrm{S}}$ with a phase shift of $\pi$ at the central region of liquid bridge (see Figs. $5 \mathrm{a}, \mathrm{b}$ and $6 \mathrm{a}, \mathrm{b}$ ), where the velocity disturbance is most vigorous. Within the other velocity characteristic mode pair (see Figs. 5c, d and $6 \mathrm{c}, \mathrm{d})$, the two modes symmetric to both $P_{\mathrm{L}}$ and $P_{\mathrm{S}}$ provide the secondary features of the velocity disturbance over the whole liquid bridge. The major modes of the temperature disturbance show the similar symmetry characteristics as the major velocity modes.

The temporal expansion coefficients $\boldsymbol{a}_{i}(\tau)$ and $\boldsymbol{b}_{i}(\tau)$ (in Eq. (3.6)) of the characteristic modes can be obtained through the direct projection of the sampled signals onto the characteristic modes. The temporal expansion coefficient profiles for the major velocity and temperature modes in the present case are shown in Fig. 7; these profiles are sinusoidal with a constant amplitude and frequency. Within the most energetic velocity mode pair, the two modes evolve with profiles of comparable amplitude and a frequency (or basic frequency) identical to that of the quasi-steady $2 P$ Marangoni oscillatory flow with a fixed phase shift of $\pi / 4$ (see Fig. 7a), the resultant flow at the central region of the liquid bridge alternates between directions parallel and antiparallel to $P_{\mathrm{S}}$ and induces a pulsating velocity disturbance of the same frequency. On the other hand, the other mode pair evolves with profiles of much smaller amplitudes and overtone frequencies. Their interaction, however, only modifies the velocity disturbances locally due to their symmetry characteristics. The aforementioned results imply that the pulsating oscillatory disturbance for the present case originates from the interior of the liquid bridge. Moreover, the contribution of the characteristic modes to the fluctuation energy is a function of the amplitude and frequency of the temporal expansion coefficient profile. The modes evolving with profiles of large amplitude and low frequency make major contributions to the fluctuation energy and thus determine the basic frequency of the resulting oscillatory disturbance. The behaviors of the temporal expansion coefficients for the temperature characteristic modes are similar, except that the profile amplitude of the dominant temperature characteristic mode overwhelms the others as expected.

\subsection{Case 2: $P r=0.01, A s=1.4, R e=6500$, and $\varepsilon=0.4$}

The saturated quasi-steady oscillatory Marangoni flow for the present case is of the (2T) type [11]. Fig. 8 shows the flow and thermal fields in a plane at $z=0.5 \mathrm{As}$ at four different instants during a quasi-steady oscillation period. The superposition of a torsional disturbance induces the backward and forward motion of the flow and thermal fields in the azimuthal direction with respect to $P_{\mathrm{L}}$. As shown in Fig. 2b, the basic flow and thermal fields, which are symmetric to both $P_{\mathrm{L}}$ and $P_{\mathrm{S}}$, are also similar to the threedimensional steady flow of $m=2$. Both the velocity and temperature disturbances are characterized by dominant

Table 2

Eigenvalues of major characteristic modes of velocity and temperature disturbances for $\operatorname{Pr}=0.01, \mathrm{As}=1.4$, and $\operatorname{Re}=6500$

\begin{tabular}{|c|c|c|c|c|}
\hline \multirow[t]{2}{*}{ Eigenfunction, $i$} & \multicolumn{2}{|l|}{ Velocity disturbance } & \multicolumn{2}{|l|}{ Temperature disturbance } \\
\hline & Normalized eigenvalue, $\lambda_{i}$ & $\begin{array}{l}\text { Contribution to fluctuation } \\
\text { energy by } \sum \lambda_{i}(\%)\end{array}$ & Normalized eigenvalue, $\lambda_{i}$ & $\begin{array}{l}\text { Contribution to fluctuation } \\
\text { energy by } \sum \lambda_{i}(\%)\end{array}$ \\
\hline 1 & 0.4879 & 48.789 & 0.7471 & 74.713 \\
\hline 2 & 0.4347 & 92.259 & 0.2034 & 95.050 \\
\hline 3 & $3.5095 \times 10^{-2}$ & 95.769 & $3.0617 \times 10^{-2}$ & 98.112 \\
\hline 4 & $3.3516 \times 10^{-2}$ & 99.120 & $1.7723 \times 10^{-2}$ & 99.884 \\
\hline 5 & $3.6923 \times 10^{-3}$ & 99.490 & $5.2236 \times 10^{-4}$ & 99.937 \\
\hline 6 & $3.5015 \times 10^{-3}$ & 99.840 & $3.5723 \times 10^{-4}$ & 99.972 \\
\hline 7 & $6.4880 \times 10^{-4}$ & 99.905 & $1.4593 \times 10^{-4}$ & 99.987 \\
\hline 8 & $6.2786 \times 10^{-4}$ & 99.967 & $5.1510 \times 10^{-5}$ & 99.992 \\
\hline 9 & $1.1525 \times 10^{-4}$ & 99.979 & $3.8252 \times 10^{-5}$ & 99.996 \\
\hline 10 & $1.1417 \times 10^{-4}$ & 99.999 & $1.4697 \times 10^{-5}$ & 99.999 \\
\hline
\end{tabular}


a

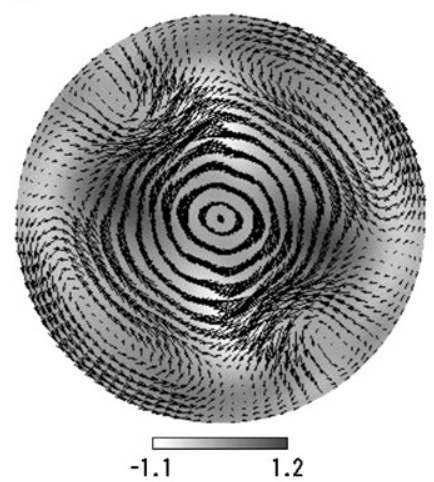

b

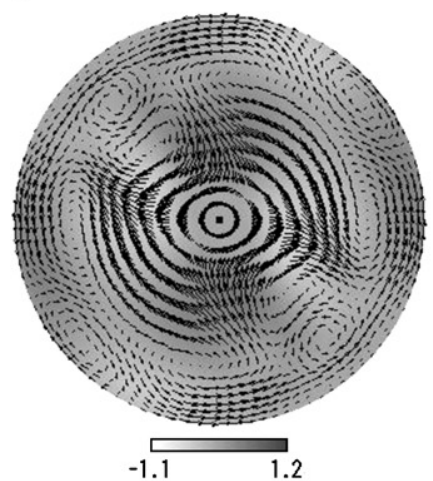

C

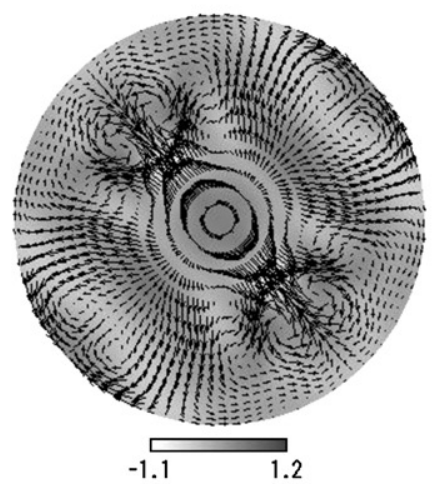

d
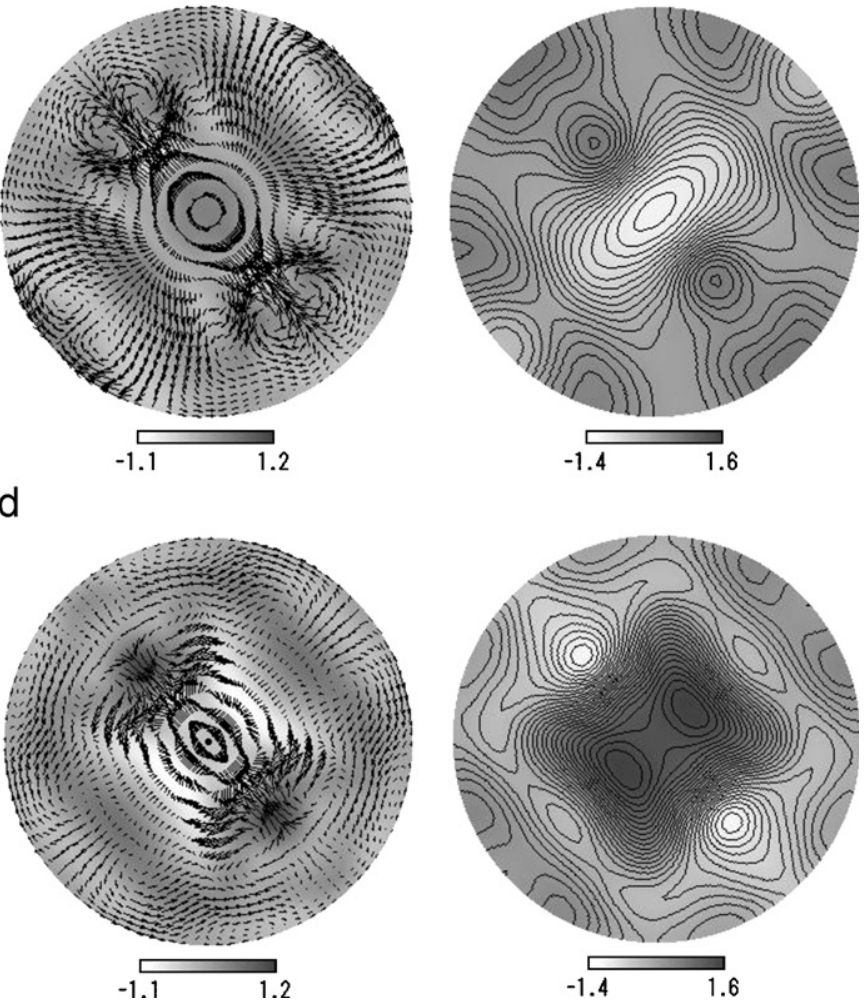

Fig. 10. Projections of the major velocity and temperature characteristic modes of the $2 T$ oscillatory flow onto the $z=0.5$ As plane (gray color in the flow-field plots denotes the axial velocity). disturbances with even azimuthal wave numbers (see Fig. 9), which are different from the aforementioned pulsating disturbance in the $(2 P)$ case. Table 2 shows the 10 largest normalized eigenvalues of the characteristic modes of the velocity disturbance and temperature disturbance obtained through the POD analysis. For the velocity disturbance, the a

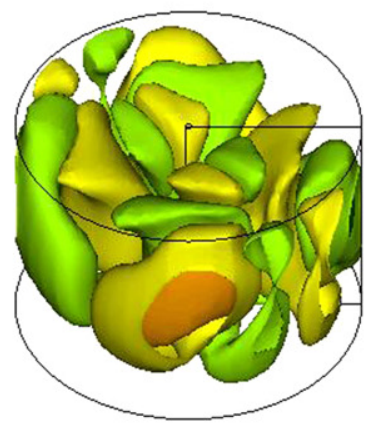

C

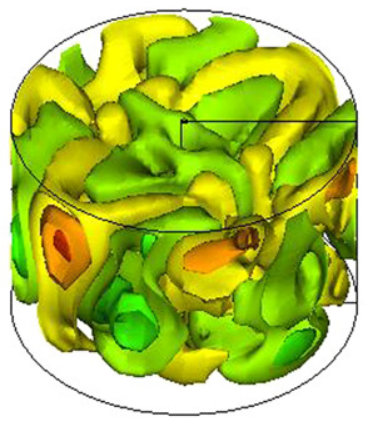

e

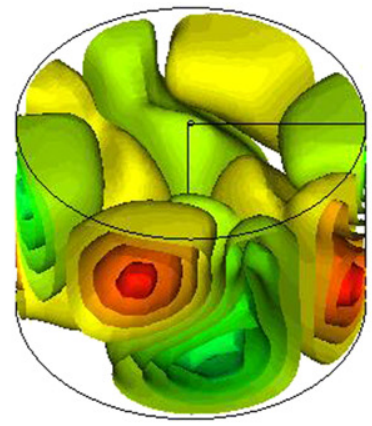

g

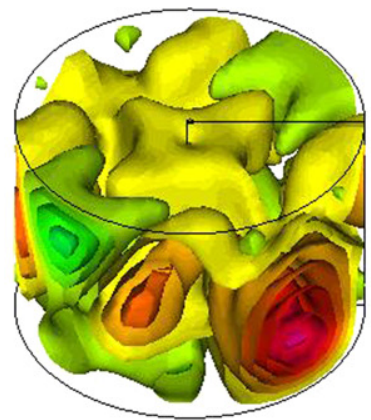

b

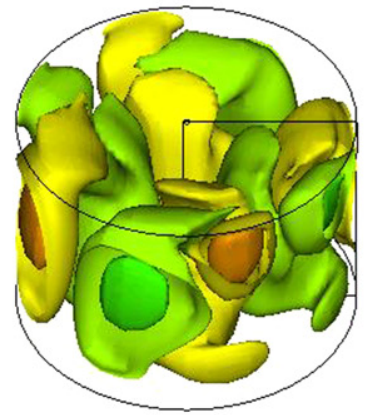

d

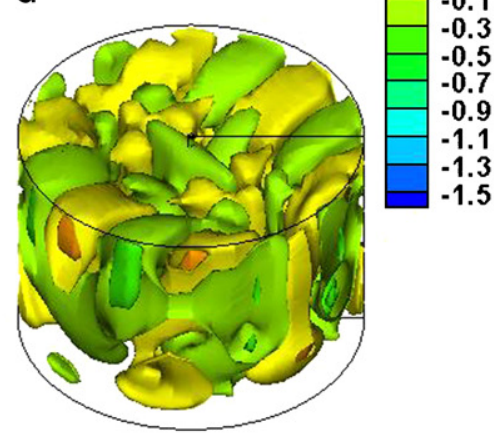

$f$

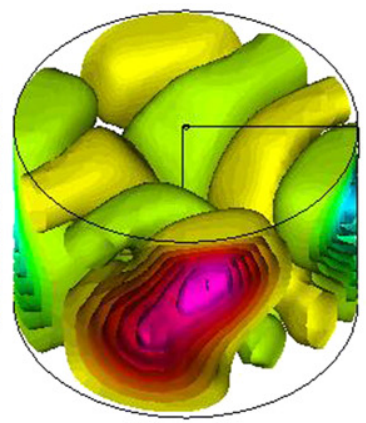

h

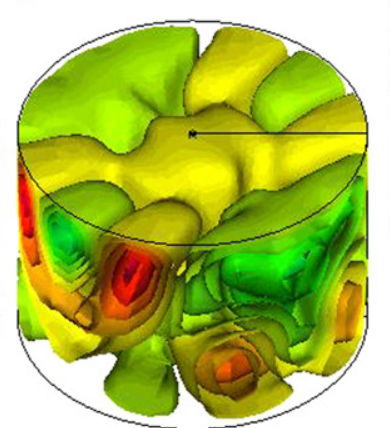

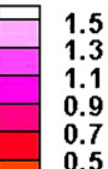

0.7

0.3

0.1

0.1

-0.3
-0.5

$-0.7$

$-1.1$
Fig. 11. Iso-surfaces of the major characteristic modes of $2 T$ oscillatory flow: (a)-(d) the velocity modes and (e)-(h) the temperature characteristic modes. 
eigenvalues form pairs, and the eigenvalues in each pair are approximately equal. Similar to the case of the $(2 P)$ oscillatory Marangoni flow, the first two pairs of characteristic modes totally capture more than $99 \%$ of the fluctuation energy, and the first pair is the most energetic which captures more than $90 \%$ of the fluctuation energy. For the temperature disturbance, the contribution to the temperature fluctuation energy of the first characteristic mode is also dominant. However, the percentage, which is about $75 \%$, is less than the dominant temperature characteristic mode in the case of the $(2 P)$ oscillatory Marangoni flow. Within the most energetic characteristic velocity mode pair (see Figs. 10a, b and $11 \mathrm{a}, \mathrm{b}$ ), each mode consists of two diagonal pairs of major vortices along $P_{\mathrm{L}}$ and $P_{\mathrm{S}}$. The vortices in each pair are antisymmetric with respect to the axis of the liquid bridge and have the same vorticity direction. The velocity at the central region of the liquid bridge has a strong component approximately perpendicular to $P_{\mathrm{L}}$ and $P_{\mathrm{S}}$. However, the vorticity directions of the vortex pairs along $P_{\mathrm{L}}$ of the two modes are opposite to each other. When the two modes evolve with temporal expansion coefficient profiles of the same basic frequency with a fixed phase shift of $\pi / 4$ (see Fig. 12), the resulting velocity component perpendicular to $P_{\mathrm{L}}$ periodically enhances and counteracts the flow circulating about the axis of the liquid bridge; the velocity component also induces the alternate expansion and contraction of the diagonal pairs of vortices in the azimuthal direction. Correspondingly, the flow field shows a torsional oscillatory motion of the same frequency with respect to $P_{\mathrm{L}}$. Within the second most energetic velocity characteristic mode pair (see Figs. 10c, d and 11c, d), the two modes symmetric to both $P_{\mathrm{L}}$ and $P_{\mathrm{S}}$ provide the secondary features of the velocity disturbance. The interaction of the two modes only modifies the velocity disturbance locally due to their symmetry with regard to both $P_{\mathrm{L}}$ and $P_{\mathrm{S}}$. The major modes of the temperature disturbance show the same symmetry characteristics as those of the velocity disturbance. Similar to the velocity disturbance, the two characteristic modes evolving with profiles of comparable amplitudes and a basic frequency identical to the $(2 T)$ oscillatory Marangoni flow make a major contribution to the temperature fluctuation energy.

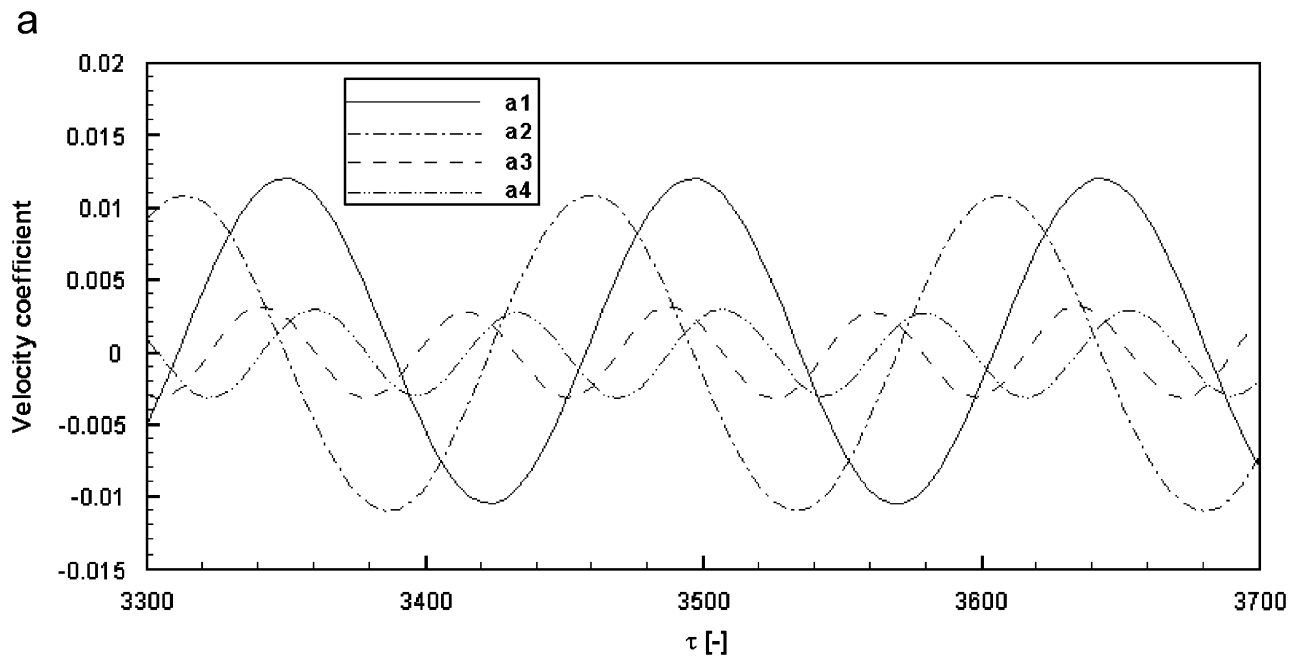

b

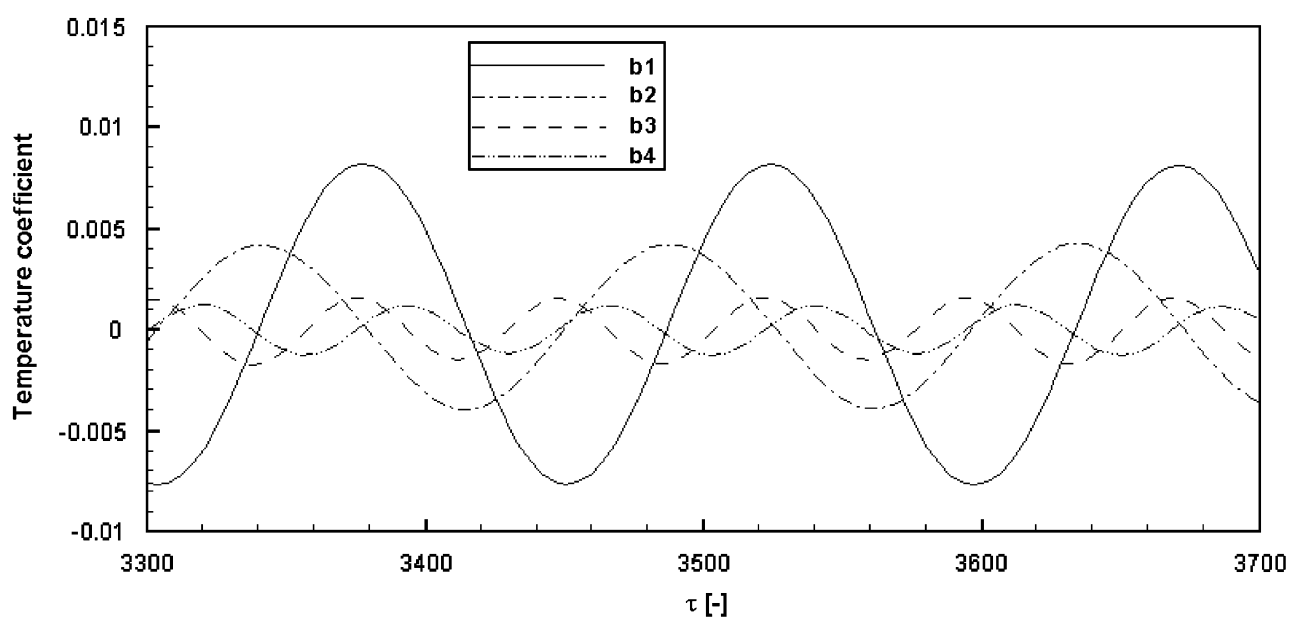

Fig. 12. Temporal expansion coefficient of the major characteristic modes for the $2 T$ oscillations: (a) velocity characteristic modes and (b) temperature characteristic modes. 
a

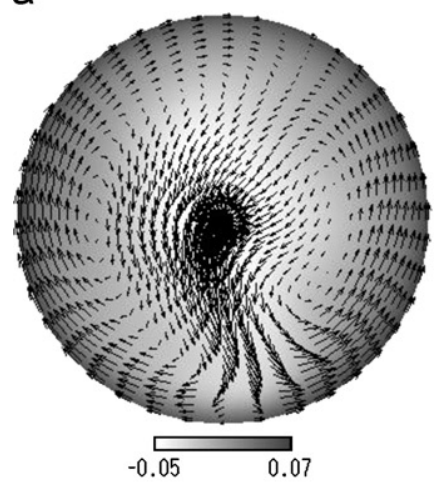

b

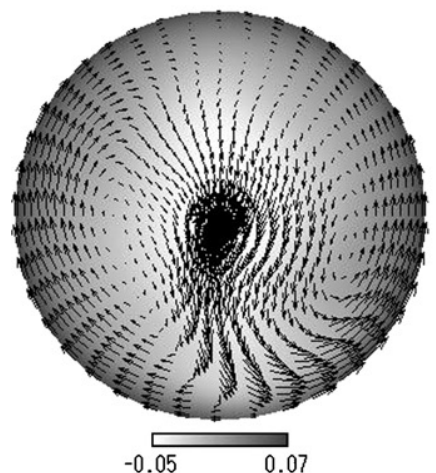

C

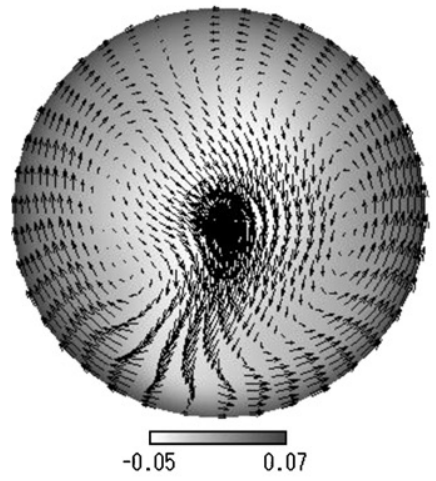

d

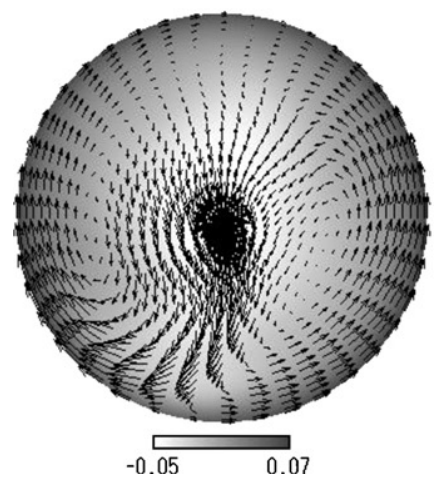

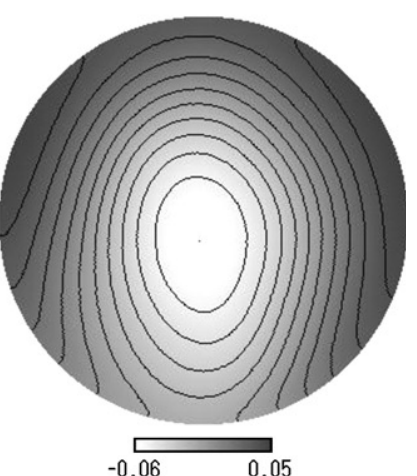
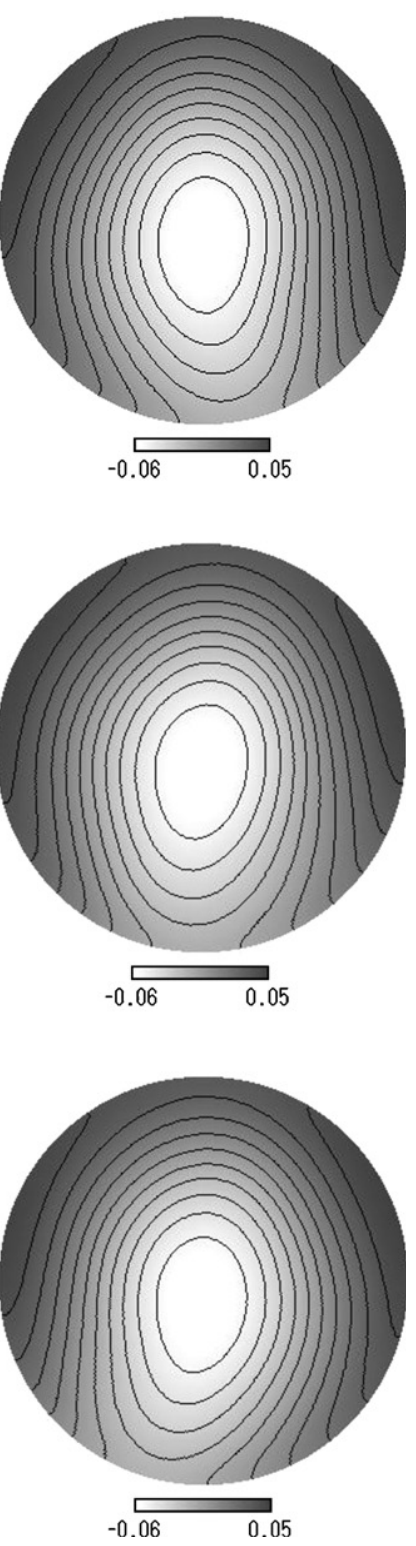

Fig. 13. Projections of flow and thermal fields of the $1 T$ oscillatory flow onto the $z=0.5 \mathrm{As}$ plane for a quasi-steady oscillation period at intervals of $\tau_{0} / 4$ (gray color in the flow-field plots denotes the axial velocity). a
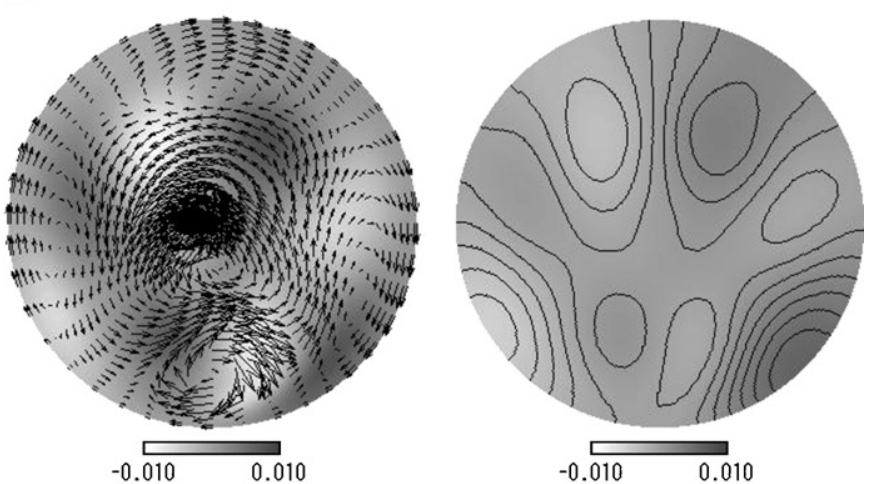

b
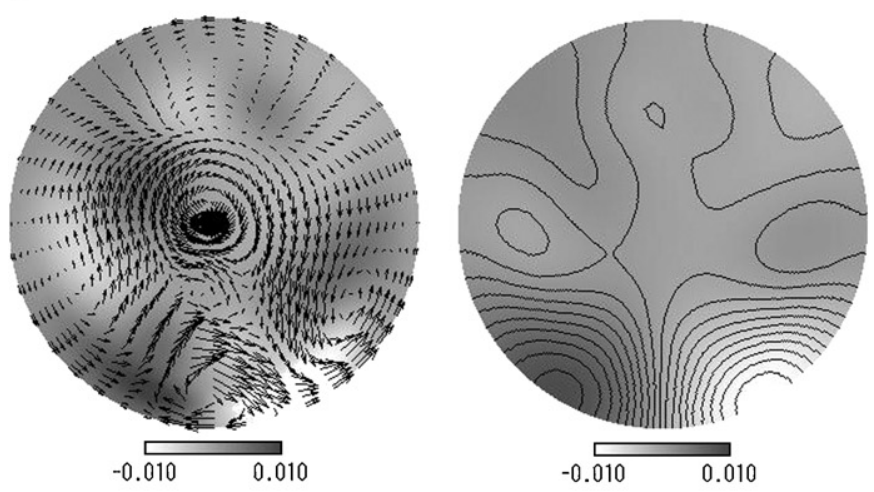

C
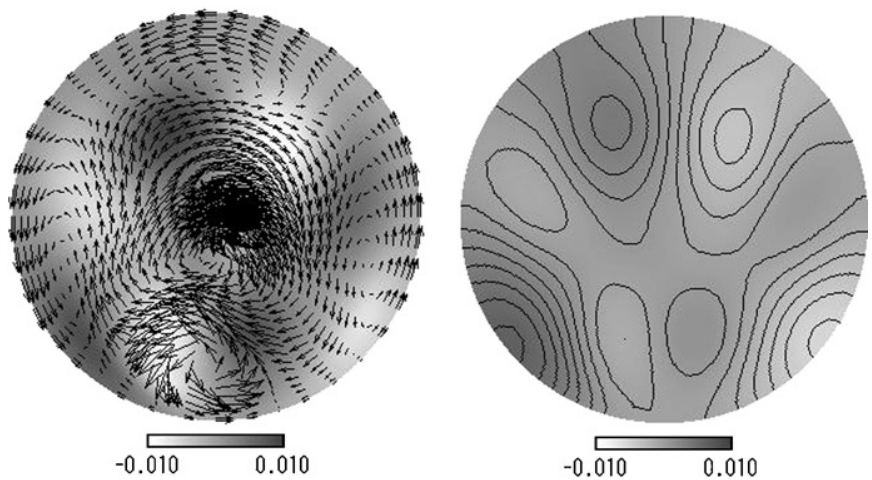

d
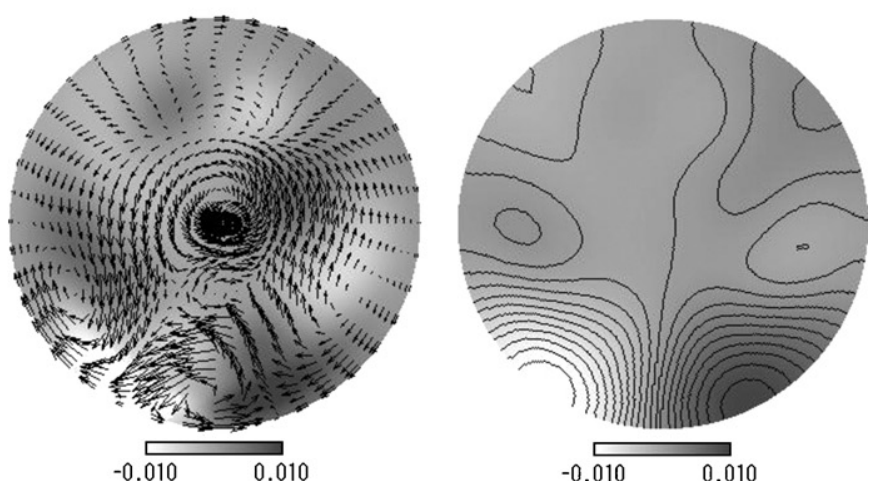

Fig. 14. Projections of velocity and temperature disturbances of the $1 T$ oscillatory flow onto the $z=0.5$ As plane for a quasi-steady oscillation period at intervals of $\tau_{0} / 4$ (gray color in the flow-field plots denotes axial velocity). 
Table 3

Eigenvalues of major characteristic modes of velocity and temperature disturbances for $P r=0.01$, As $=2.0$, and $R e=3500$

\begin{tabular}{|c|c|c|c|c|}
\hline Eigenfunction, $i$ & \multicolumn{2}{|l|}{ Velocity disturbance } & \multicolumn{2}{|l|}{ Temperature disturbance } \\
\hline 1 & 0.5059 & 50.587 & 0.7103 & 71.030 \\
\hline 2 & 0.4569 & 96.274 & 0.2775 & 98.783 \\
\hline 3 & $1.7682 \times 10^{-2}$ & 98.042 & $6.3910 \times 10^{-3}$ & 99.421 \\
\hline 4 & $1.7184 \times 10^{-2}$ & 99.761 & $5.5394 \times 10^{-3}$ & 99.976 \\
\hline 7 & $9.3990 \times 10^{-5}$ & 99.985 & $4.3849 \times 10^{-5}$ & 99.998 \\
\hline 8 & $9.3291 \times 10^{-5}$ & 99.999 & $2.9853 \times 10^{-6}$ & 99.999 \\
\hline
\end{tabular}

4.3. Case 3: $\operatorname{Pr}=0.01, A s=2.0, \operatorname{Re}=3500$, and $\varepsilon=0.22$

The saturated quasi-steady oscillatory Marangoni flow in the present case is of the (1T) type. Fig. 13 shows the flow and thermal fields in a plane at $z=0.5 \mathrm{As}$ at four different instants during an oscillation period. The torsional disturbance in the present case induces an oscillatory motion in the flow and thermal fields in the azimuthal direction with respect to the symmetry plane of the basic flow and thermal fields of $m=1$ (denoted as $P_{\mathrm{SY}}$ ) (see Fig. 2c). Fig. 14 shows the time evolution of velocity and temperature disturbances over an oscillation period. The flow circulating about the axis of the liquid bridge periodically alters its orientation between anticlockwise and clockwise directions; it is accompanied by an intense vortex cell at the cold plume altering the flow orientation in the reverse order. Table 3 shows the eight largest normalized eigenvalues of the characteristic modes of the velocity disturbance and temperature disturbance. For the velocity disturbance, the first two pairs of characteristic modes totally capture more than $99 \%$ of the fluctuation energy, and the first pair is the most energetic. For the temperature disturbance, the first characteristic mode makes the dominant contribution to the temperature fluctuation energy. Within the most energetic velocity characteristic mode pair (see Figs. 15a, b and 16a, b), each mode consists of two diagonal pairs of major vortices along $P_{\mathrm{SY}}$ and antisymmetric to $P_{\mathrm{SY}}$, and the velocity at the central region has a strong component circulating about the axis of liquid bridge. However, for the pairs of vortices along $P_{\mathrm{SY}}$, only the intense vortices in the cold plume have opposite vorticity directions, which is different from the $(2 T)$ oscillatory Marangoni flow. When the two modes evolve with temporal expansion coefficient profiles of the basic frequency with a fixed phase shift (see Fig. 17), the resulting vortices in the cold plume, which alter the flow orientation between anticlockwise and clockwise directions alternately, enhance and reduce the flow periodically circulating about the axis of the liquid bridge. The cold plume vortices also induce the alternate expansion and contraction of the vortex pair antisymmetric to $P_{\mathrm{SY}}$. Correspondingly, the flow field shows a torsional oscillatory motion of the same frequency with respect to the half of $P_{\mathrm{SY}}$ in the cold plume. The other two major velocity characteristic modes (see Figs. 15c, d and 16c, d) are both symmetric to $P_{\mathrm{SY}}$. Their interaction only modifies the velocity disturbance locally.

\subsection{Dependency on the signal length and the number of snapshots}

In the present study, the sampled time evolution signals of the flow and thermal fields covered three periods of the quasi-steady oscillations to show the periodic evolutions of the temporal expansion coefficients $\boldsymbol{a}_{i}(\tau)$ and $\boldsymbol{b}_{i}(\tau)$. It is noted that for purely quasi-steady oscillation, the signal length of one quasi-steady period is enough. Moreover, we collected different samples from the same signal by changing the number of snapshot, i.e., $N=240,120$, and 60 . It is found that they gave the similar POD results except for $N=60$. Thus, a sufficient small time interval between snapshots is required for accuracy of the POD results. However, it is unnecessary to strongly increase the number of snapshots to avoid the increasing computation task.

\section{Conclusions}

In order to visualize the details of the spatio-temporal structures of three-dimensional oscillatory disturbances, POD analysis using the method of snapshots was applied to the oscillatory Marangoni flows in low- $P r(P r=0.01)$ liquid bridges of $\mathrm{As}=1.0, \quad R e=8000 ; \quad \mathrm{As}=1.4$, $R e=6500$; and As $=2.0, R e=3500$. At slightly supercritical conditions, the saturated oscillatory Marangoni flows are quasi-steady and correspond to $(2 P),(2 T)$, and (1T) types. The POD results reveal that the oscillatory flow and temperature disturbances can be decomposed into a series of characteristic modes (eigenfunctions). For the Marangoni flow of different oscillatory types, the characteristic modes of velocity and temperature disturbances 
a
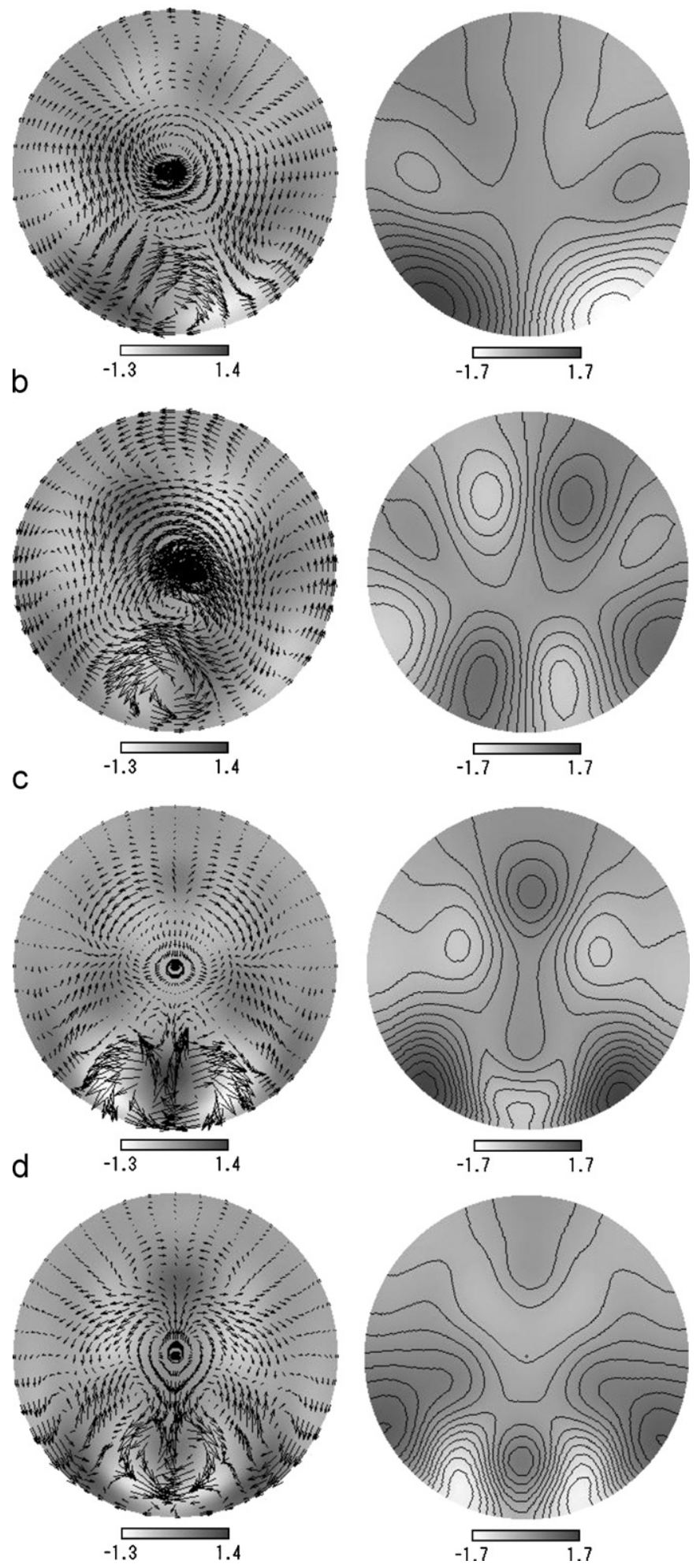

Fig. 15. Projections of the major velocity and temperature characteristic modes of the $1 T$ oscillatory flow onto the $z=0.5 \mathrm{As}$ plane (gray color in the flow-field plots denotes axial velocity).

have common features. Among the characteristic modes, only several modes with large eigenvalues, i.e., major modes, are important; these capture more than $99 \%$ of the a

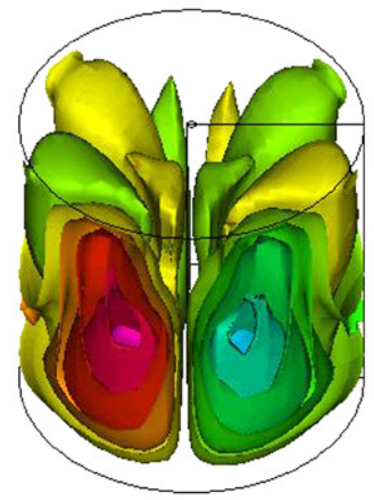

C

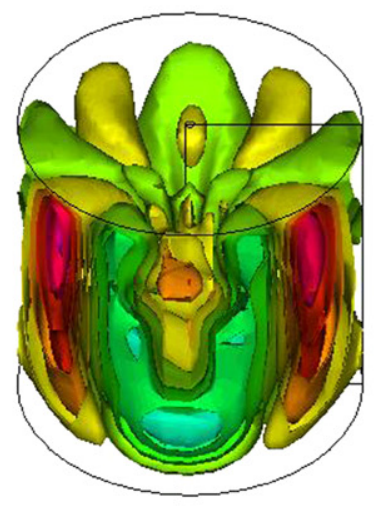

e

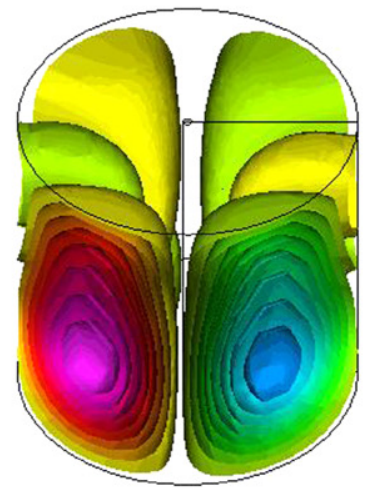

g

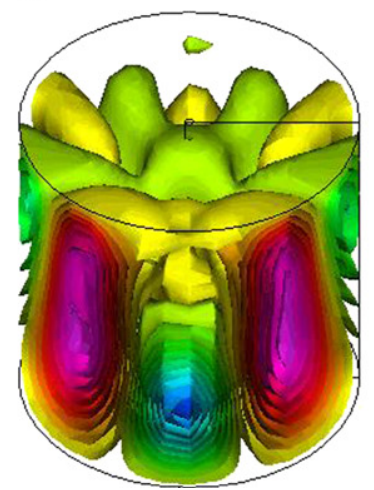

b

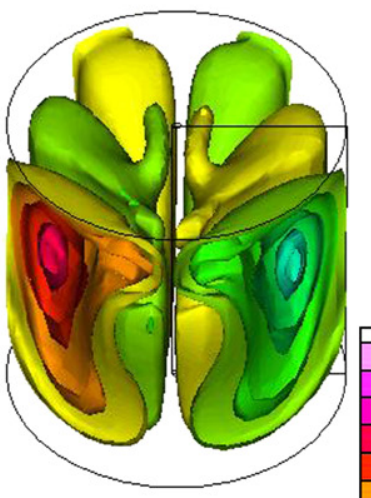

d

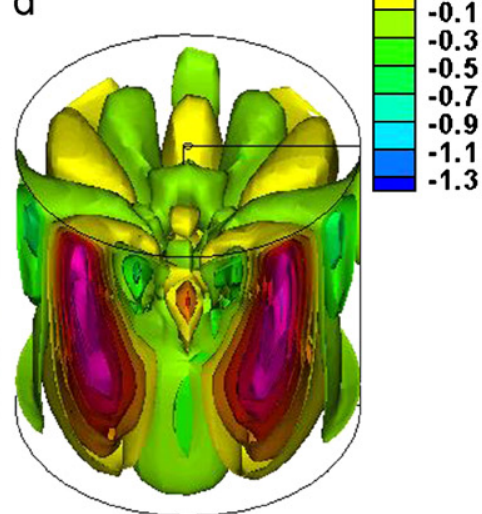

f

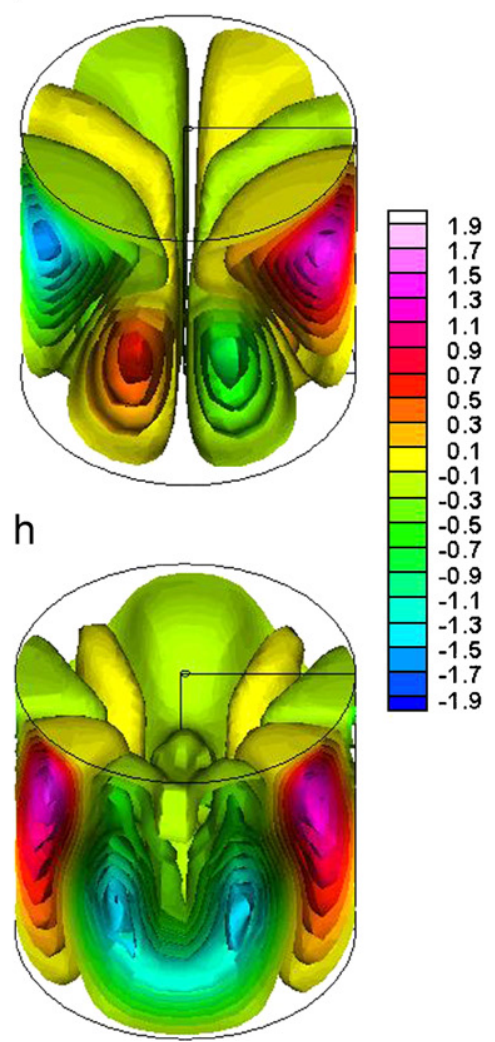

Fig. 16. Iso-surfaces of the major characteristic modes of $1 T$ oscillatory flow: (a)-(d) the velocity characteristic modes and (e)-(h) the major temperature modes. 
a

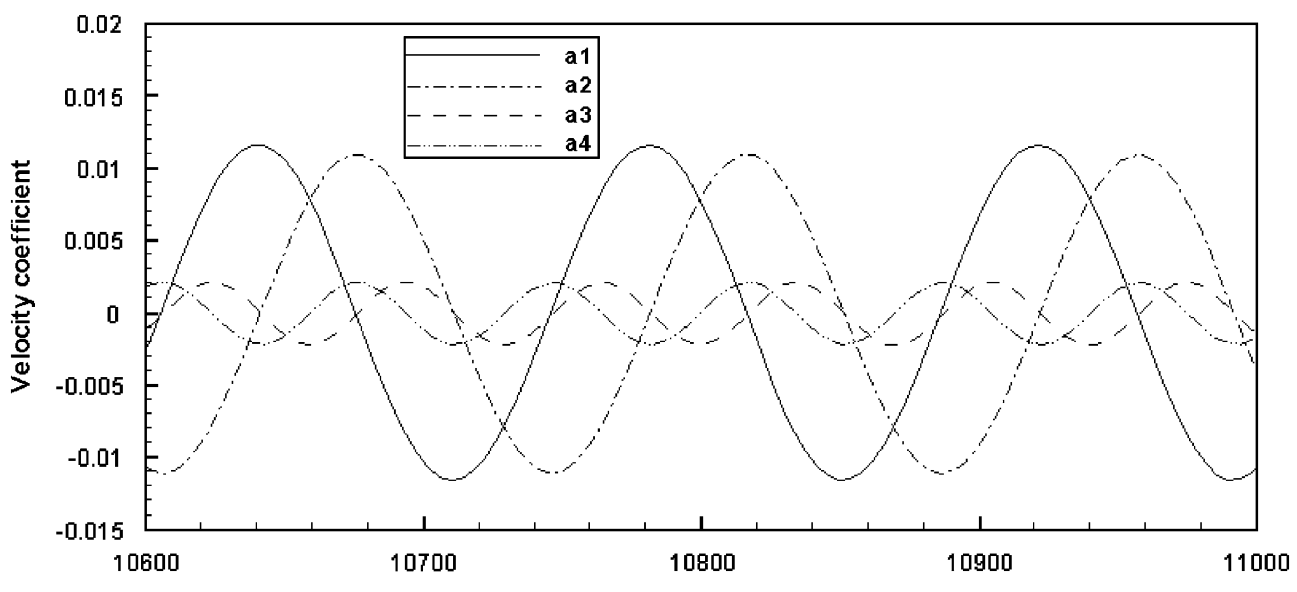

b

$\tau[-]$

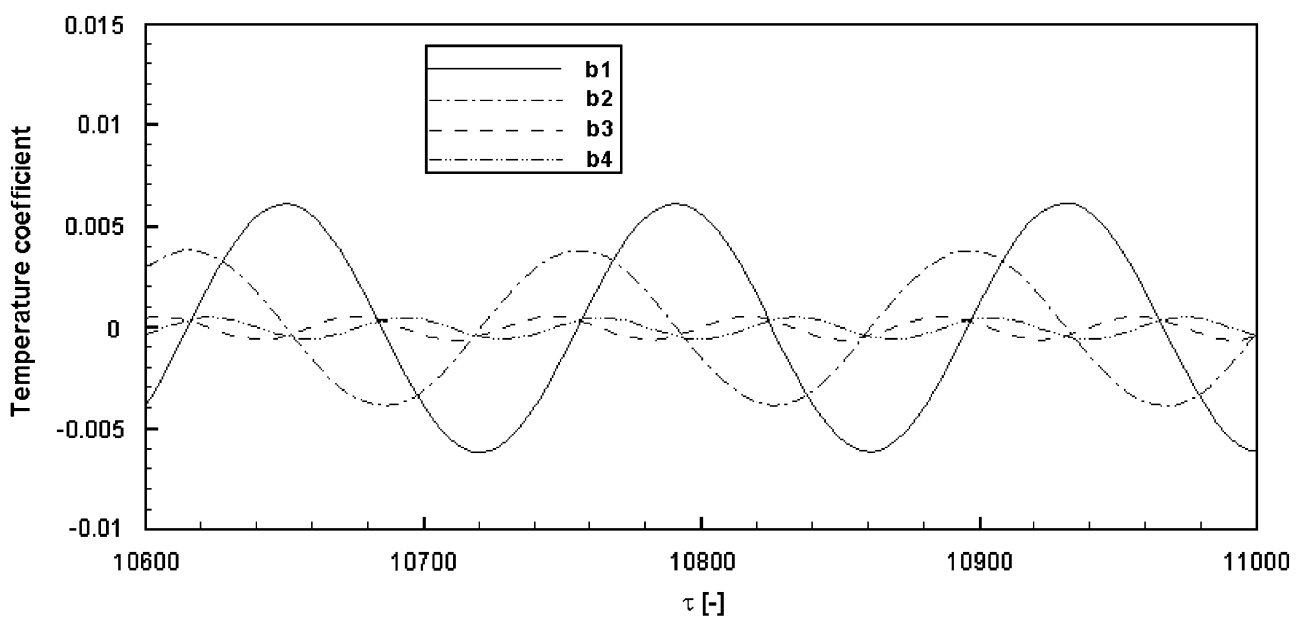

Fig. 17. . Temporal expansion coefficients of the major characteristic modes for the $1 T$ oscillations: (a) velocity characteristic modes and (b) temperature characteristic modes.

fluctuation energy. The major characteristic modes evolve with the temporal expansion coefficient profiles of large amplitude and low frequency make crucial contributions to the fluctuation energy, and thus determine the basic frequency of the resulting oscillatory flow. Moreover, the spatio-temporal structure of the oscillatory disturbance reflects the spatial characteristics of the most energetic velocity characteristic mode pair, which are unique for different oscillation types. It also implies that the oscillatory disturbance, which is hydrodynamic in nature, primarily originates from the interior of the liquid bridge. The interaction of other major velocity modes only modifies the disturbances locally due to their symmetry characteristics, i.e., the secondary spatio-temporal structures of the disturbance. To verify the conclusions, summations of the most energetic velocity and temperature characteristic modes with their temporal expansion coefficients are adopted to reproduce the original oscillatory velocity and temperature disturbances, and the results are in a good agreement.

\section{References}

[1] H.C. Kuhlmann, Thermocapillary Convection in Models of Crystal Growth, Springer, New York, 1999.

[2] A. Croell, Th. Kaiser, A. Schueizer, et al., J. Crystal Growth 191 (1998) 365.

[3] S. Nakamura, T. Hibiya, K. Kakimoto, N. Imaishi, S. Nishizawa, A. Hirata, K. Mukai, S. Yoda, T.S. Morita, J. Jpn. Soc. Microgravity Appl. 16 (1999) 99.

[4] R. Rupp, G. Mueller, G. Neumann, J. Crystal Growth 97 (1989) 34.

[5] M. Levenstam, G. Amberg, C. Winkler, Phys. Fluids 13 (4) (2001) 807.

[6] M. Levenstam, G. Amberg, J. Fluid Mech. 297 (1995) 357.

[7] J. Leypoldt, H.C. Kuhlmann, H.J. Rath, J. Fluid Mech. 414 (2000) 285.

[8] M. Lappa, R. Savino, R. Monti, Int. J. Heat Mass Transfer 44 (2001) 1983.

[9] V. Shevtsova, J. Crystal Growth 280 (2005) 632.

[10] N. Imaishi, S. Yasuhiro, T. Sato, S. Yoda, in: Proceedings of Materials Research in Low Gravity 2 SPIE International Symposium, vol. 3792, 1999, p. 344. 
[11] N. Imaishi, S. Yasuhiro, T. Sato, S. Yoda, in: Proceedings of the Fourth JSME-KSME Thermal Engineering Conference, 1-6 October 2000, Kobe, Japan, p. 272.

[12] S. Yasuhiro, T. Sato, N. Imaishi, S. Yoda, Space Forum 6 (2000) 39.

[13] N. Imaishi, S. Yasuhiro, Y. Akiyama, S. Yoda, J. Crystal Growth 230 (2001) 164.

[14] J.L. Lumley, in: A.M. Yaglom, V.I. Tatarski (Eds.), Atmospheric Turbulence and Radio Wave Propagation, Nauka, Moscow, 1967, p. 166.
[15] L. Sirvoich, Quart. Appl. Math. XLV (1987) 561.

[16] L. Sirvoich, Fluid Dyn. Res. 8 (1991) 85.

[17] G. Berkooz, P. Holmes, J. L Lumely, Annu. Rev. Fluid Mech. 25 (1993) 539

[18] H. Gunes, A. Liakopoulos, R.A. Sahan, Theor. Comput. Fluid Dyn. 9 (1997) 1.

[19] R.A. Sahan, A. Liakopoulos, H. Gunes, Phys. Fluids 9 (1997) 551.

[20] H. Gunes, Fluid Dyn. Res. 30 (2002) 1.

[21] L. Sirvoich, Physica D 37 (1989) 126. 